\title{
Adaptive Optimal Stochastic Trajectory Planning and Control (AOSTPC) for Robots
}

\author{
K. Marti \\ Federal Armed Forces University \\ Aero-Space Engineering and Technology \\ D - 85577 Neubiberg/München, Germany \\ E-mail: kurt.marti@unibw-muenchen.de
}

\section{Introduction}

Modeling a robot (industrial or service robot) by its dynamic equation, being a system of second order differential equations for the robot or configuration coordinates $q=\left(q_{1}, \ldots, q_{n}\right)^{\prime}$ (rotation angles in case of revolute links, length of translations in case of prismatic links), and the kinematic equation, relating the space $\{q\}$ of robot coordinates to the work space $\{x\}$ of the robot, one meets $[4,29$, $35,38,40,42]$ several model parameters, such as length of links, $l_{i}(m)$, location of center of gravity of links, $l_{c i}(m)$, mass of links, $m_{i}(k g)$, payload $(N)$, moments of inertia about centroid, $I_{i}\left(\mathrm{kgm}^{2}\right)$, (Coulomb-)friction coefficients, $R_{i j 0}(N)$, etc.. Let $p_{D}, p_{K}$ denote the vector of model parameters contained in the dynamic, kinematic equation, respectively. A further vector $p_{C}$ of model parameters occurs in the formulation of several constraints, especially initial and terminal conditions, control and state constraints of the robot, as e.g. maximum, minimum torques or forces in the links, bounds for the position, maximum joint, path velocities; moreover, certain parameters $p_{J}$, e.g. cost factors, may occur also in the objective (performance, goal) functional J.

Due to stochastic variations of the material, manufacturing errors, measurement (identification) errors, stochastic variations of the workspace environment, as e.g. stochastic uncertainty of the payload, randomly changing obstacles, errors in the selection of appropriate bounds for the moments, forces, resp., in the links, 
for the position and path velocities, errors in the selection of random cost factors, modelling errors, disturbances, etc., the total vector

$$
p=\left(\begin{array}{c}
p_{D} \\
p_{K} \\
p_{C} \\
p_{J}
\end{array}\right)
$$

of model parameters is not a given fixed quantity, but must be represented by a random vector

$$
p=p(\omega), \quad \omega \in(\Omega, \mathcal{A}, P)
$$

on a certain probability space $(\Omega, \mathcal{A}, P)$, see $[3,13,27,38,44]$.

Having to control a robotic or more general dynamical system, the control law $u=u(t)$, is represented usually by the sum

$$
u(t):=u^{(0)}(t)+\Delta u(t), t_{0} \leq t \leq t_{f},
$$

of a feedforward control (open-loop-control) $u_{0}(t), t_{0} \leq t \leq t_{f}$, and an on-line local control correction (feedback control) $\Delta u(t)$.

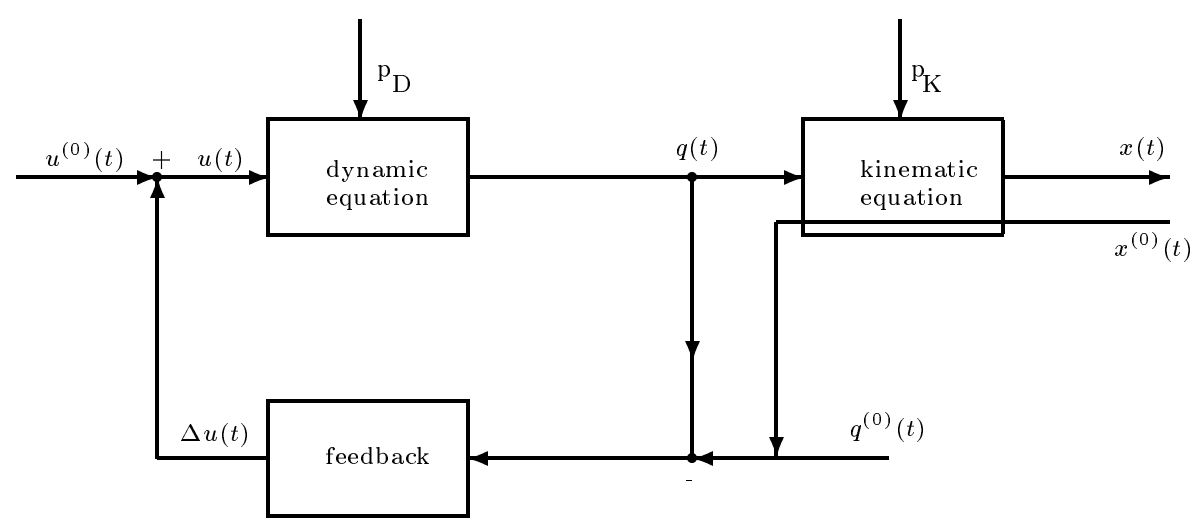

Fig. 1.1 Control of dynamic systems (robots)

In actual engineering practice $[18,28,30,45]$, the feedforward control $u^{(0)}(t)$ is determined off-line based on a certain reference trajectory $q^{(0)}(t), t_{0} \leq t \leq t_{f}$, in configuration space, and by replacing the unknown parameter vector $p$ by a certain vector $p^{(0)}$ of nominal parameter values, as e.g. the expectation $p^{(0)}:=$ $\bar{p}=E p(\omega)$. The resulting, and usually increasing deviation of the actual position and velocity of the robot from the prescribed values, caused by the deviation of the actual parameter values $p(\omega)$ from the chosen nominal values $p^{(0)}$, must be 
compensated by an on-line control correction $\Delta u(t)$ at each time point $t>t_{0}$. This requires usually extensive on-line state observations (measurements) and feedback control actions.

In order to determine a more reliable reference path $q=q(t), t_{0} \leq t \leq t_{f}$, in configuration space, being robust with respect to stochastic parameter variations, the a priori information (given by certain moments or parameters of the probability distribution of $p(\cdot))$ about the random variations of the vector $p(\omega)$ of model parameters of the robot and its working environment are taken into account already at the planning phase. Thus, instead of solving a deterministic path planning problem with a fixed nominal parameter vector $p^{(0)}$, here, an optimal velocity profile $\beta^{(0)}, s_{0} \leq s \leq s_{f}$, and - in case of point-topoint control problems - also an optimal geometric path $\tilde{q}^{(0)}(s), s_{0} \leq s \leq s_{0}$, in configuration space is determined by using a stochastic optimization approach. By means of $\beta^{(0)}(s)$ and $\tilde{q}^{(0)}(s), s_{0} \leq s \leq s_{f}$, we find then a more reliable, robust reference path $q^{(0)}(t), t_{0} \leq t \leq t_{f}^{(0)}$, in configuration space. Applying now the so-called "inverse dynamics approach" [1,4,14], more reliable, robust open-loop controls $u^{(0)}(t), t_{0} \leq t \leq t_{f}^{(0)}$, are obtained. Moreover, by linearization of the dynamic equation of the robot in a neighborhood of $\left(u^{(0)}(t), q^{(0)}(t), E\left(p_{M}(\omega) \mid \mathcal{A}_{t_{0}}\right)\right), t \geq t_{0}$, where $\mathcal{A}_{t_{0}}$ denotes the $\sigma$-algebra of informations up to the initial time point $t_{0}$, a control correction $\Delta u^{(0)}(t), t \geq t_{0}$, is obtained which is related to the so-called feedback linearization of a system $[4,14,32,40]$.

Since at later time instants (main correction time points) $t_{j}$,

$$
t_{0}<t_{1}<t_{2}<\ldots<t_{j-1}<t_{j}<\ldots
$$

further information on the parameters of the control system and its environment are available, e.g., by process observation, identification, calibration procedures etc., improvements $q^{(j)}(t), u^{(j)}(t), \Delta u^{(j)}(t), t \geq t_{j}, j=$ $1,2, \ldots$, of the initial (preceding) reference trajectory $q^{(0)}(t)\left(q^{(j-1)}(t)\right)$, open loop control $u^{(0)}(t)\left(u^{(j-1)}(t)\right)$, and local control correction (feedback control) $\Delta u^{(0)}(t)\left(\Delta u^{(j-1)}(t)\right)$ can be determined by replanning, i.e., by optimal stochastic trajectory planning (OSTP) for the remaining time interval $t \geq t_{j}, j=1,2, \ldots$, 
and by using the information $\mathcal{A}_{t_{j}}$ on the robot and its working environment available up to the time point $t_{j}>t_{0}, j=1,2, \ldots$.

\section{Optimal Trajectory Planning for Robots}

According to $[4,29,38]$, the dynamic equation for a robot is given by the following system of second order differential equations

$$
M\left(p_{D}, q(t)\right) \ddot{q}(t)+h\left(p_{D}, q(t), \dot{q}(t)\right)=u(t), t \geq t_{0},
$$

for the $n$-vector $q=q(t)$ of the robot or configuration coordinates $q_{1}, q_{2}, \ldots, q_{n}$. Here, $M=M\left(p_{D}, q\right)$ denotes the $n \times n$ inertia (or mass) matrix, and the vector function $h=h\left(p_{D}, q, \dot{q}\right)$ is given by

$$
h\left(p_{D}, q, \dot{q}\right):=C\left(p_{D}, q, \dot{q}\right) \dot{q}+F_{R}\left(p_{D}, q, \dot{q}\right)+G\left(p_{D}, q\right)
$$

where $C\left(p_{D}, q, \dot{q}\right)=C\left(p_{D}, q\right) \dot{q}$, and $C\left(p_{D}, q\right)=\left(C_{i j k}\left(p_{D}, q\right)\right)_{1 \leq i, j, k \leq n}$ is the tensor of Coriolis and centrifugal terms, $F_{R}=F_{R}\left(p_{D}, q, \dot{q}\right)$ denotes the vector of frictional forces and $G=G\left(p_{D}, q\right)$ is the vector of gravitational forces; moreover, $u=u(t)$ is the vector of controls, i.e., the vector of torques/forces in the joints of the robot. Standard representations of the friction term $F_{R}$ are given $[4,18,38]$ by

$$
\begin{aligned}
& F_{R}\left(p_{D}, q, \dot{q}\right):=R_{v}\left(p_{D}, q\right) \dot{q}, \\
& F_{R}\left(p_{D}, q, \dot{q}\right):=R\left(p_{D}, q\right) \operatorname{sgn}(\dot{q}),
\end{aligned}
$$

where $\operatorname{sgn}(\dot{q}):=\left(\operatorname{sgn}\left(\dot{q}_{1}\right), \ldots, \operatorname{sgn}\left(\dot{q}_{n}\right)\right)^{\prime}$. In the first case $(4 \mathrm{a}), R_{v}=R_{v}\left(p_{D}, q\right)$ is the viscous friction matrix, and in the Coulomb approach (4d), $R=R\left(p_{D}, q\right)=$ $\left(R_{i}(p, q) \delta_{i j}\right)$ is a diagonal matrix.

Remark 2.1. Inverse dynamics. Reading the dynamic equation (4a) from the left to the right hand side, hence, by inverse dynamics $[1,4,14]$, the control function $u=u(t)$ may be described in terms of the trajectory $q=q(t)$ in configuration space.

The relationship between the so-called configuration space $\{q\}$ of robot coordinates $q=\left(q_{1}, \ldots, q_{n}\right)^{\prime}$ and the work space $\{x\}$ of world coordinates (position and 
orientation of the end-effector) $x=\left(x_{1}, \ldots, x_{n}\right)^{\prime}$ is represented by the kinematic equation

$$
x=T\left(p_{K}, q\right) .
$$

As mentioned already in the introduction, $p_{D}, p_{K}$, denote the vectors of dynamic, kinematic parameters arising in the dynamic and kinematic equation (4a-d), (5).

Remark 2.2. Linear parametrization of robots. Note that the parametrization of a robot can be chosen, cf. $[1,4,14]$, such that the dynamic and kinematic equation depend linearly on the parameter vectors $p_{D}, p_{K}$.

The objective of optimal trajectory planning is to determine $[6,7,18,28,30,39,43]$ a control function $u=u(t), t \geq t_{0}$, such that the cost functional

$$
J(u(\cdot)):=\int_{t_{0}}^{t_{f}} L\left(t, p_{J}, q(t), \dot{q}(t), u(t)\right) d t+\phi\left(t_{f}, p_{J}, q\left(t_{f}\right), \dot{q}\left(t_{f}\right)\right)
$$

is minimized, where the terminal time $t_{f}$ may be given explicitly or implicitly, as e.g. in minimum-time problems. Standard examples are, see e.g. [29]: a) $\phi=0, L=1$ (minimum time), b) $\phi=0, L=$ sum of potential, translatory and rotational energy of the robot (minimum energy), c) $\phi=0, L=\sum_{i=1}^{n}\left(\dot{q}_{i}(t) u_{i}(t)\right)^{2}$ (minimum fuel consumption), d) $\phi=0, L=\sum_{i=1}^{n}\left(u_{i}(t)\right)^{2}$ (minimum force and moment).

Furthermore, an optimal control function $u^{*}=u^{*}(t)$ and the related optimal trajectory $q^{*}=q^{*}(t), t \geq t_{0}$, in configuration space must satisfy the dynamic equation (4a-d) and the following constraints $[6,7,9,28]$ :

i) The initial conditions

$$
q\left(t_{0}\right)=\mathbf{q}_{0}(\omega), \dot{q}\left(t_{0}\right)=\dot{\mathbf{q}}_{0}(\omega)
$$

Note that by means of the kinematic equation (5), the initial state $\left(\mathbf{q}_{0}, \dot{\mathbf{q}}_{0}\right)$ in configuration space can be represented by the initial state $\left(\mathbf{x}_{0}(\omega), \dot{\mathbf{x}}_{\mathbf{0}}(\omega)\right)$ in work space.

ii) The terminal conditions

$$
\psi\left(t_{f}, p, q\left(t_{f}\right), \dot{q}\left(t_{f}\right)\right)=0
$$


e.g.

$$
q\left(t_{f}\right)=q_{f}(\omega), \dot{q}\left(t_{f}\right)=\dot{q}_{f}(\omega)
$$

Again, by means of $(5),\left(q_{f}, \dot{q}_{f}\right)$ may be described in terms of the final state $\left(x_{f}, \dot{x}_{f}\right)$ in work space.

Note that more general boundary conditions of this type may occur at some intermediate time points $t_{0}<\tau_{1}<\tau_{2}<\ldots<\tau_{r}<t_{f}$.

iii) Control constraints

$$
\begin{aligned}
u^{\min }(t, p) \leq u(t) & \leq u^{\max }(t, p), t_{0} \leq t \leq t_{f} \\
g_{I}(t, p, q(t), \dot{q}(t), u(t)) & \leq 0, t_{0} \leq t \leq t_{f} \\
g_{I I}(t, p, q(t), \dot{q}(t), u(t)) & =0, t_{0} \leq t \leq t_{f} .
\end{aligned}
$$

iv) State constraints

$$
\begin{gathered}
S_{I}(t, p, q(t), \dot{q}(t)) \leq 0, t_{0} \leq t \leq t_{f} \\
S_{I I}(t, p, q(t), \dot{q}(t))=0, t_{0} \leq t \leq t_{f} .
\end{gathered}
$$

Using the kinematic equation (5), different types of obstacles in the work space can be described by (time-invariant) state constraints of the type $(9 \mathrm{a}, \mathrm{b})$. In robotics [28] often the following state constraints are used:

$$
\begin{aligned}
& q_{\min }\left(p_{C}\right) \leq q(t) \leq q_{\max }\left(p_{C}\right), t_{0} \leq t \leq t_{f} \\
& \dot{q}_{\min }\left(p_{C}\right) \leq \dot{q}(t) \leq \dot{q}_{\max }\left(p_{C}\right), t_{0} \leq t \leq t_{p},
\end{aligned}
$$

with certain vectors $q_{\min }, q_{\max }, \dot{q}_{\min }, \dot{q}_{\max }$ of (random) bounds.

A special constraint of the type (9b) occurs if the trajectory in work space

$$
x(t):=T\left(p_{K}, q(t)\right)
$$

should follow as precise as possible a geometric path in work space

$$
x_{e}=x_{e}\left(p_{x}, s\right), x_{0} \leq s \leq s_{f}
$$

which is known up to a certain random parameter vector $p_{x}=p_{x}(\omega)$, which is added then to the total vector $p$ of model parameters, cf. $(1 \mathrm{a}, \mathrm{b})$. 
Remark 2.3. In the following we suppose that the functions $M, h, L, \phi$ and $T$ arising in (4a-d), (5), (6) as well as the functions $\psi, g_{I}, g_{I I}, S_{I}, S_{I I}$ arising in the constraints (7b-9b) are sufficiently smooth.

\section{Problem transformation}

Since the terminal time $t_{f}$ may be given explicitly or implicitly, the trajectory $q(\cdot)$ in configuration space may have a varying domain $\left[t_{0}, t_{f}\right]$. Hence, in order to work with a given fixed domain of the unknown functions, the reference trajectory $q=q(t), t \geq t_{0}$, in configuration space is represented, cf. [18,28], by

$$
q(t):=q_{e}(s(t)), t \geq t_{0}
$$

where

$$
s=s(t), t_{0} \leq t \leq t_{f}
$$

is a strictly monotonous increasing transformation from the possibly varying time domain $\left[t_{0}, t_{f}\right]$ into a given fixed parameter interval $\left[s_{0}, s_{f}\right]$. E.g., $s \in\left[s_{0}, s_{f}\right]$ may be the path parameter of a given path in work space, cf. (11). Moreover,

$$
q_{e}=q_{e}(s), s_{0} \leq s \leq s_{f},
$$

denotes the so-called geometric path in configuration space.

Remark 3.1. In many more complicated industrial robot tasks such as grinding, welding, driving around difficult obstacles, complex assembly, etc., the geometric path $q_{e}(\cdot)$ in configuration space is predetermined off-line $[8,15,16]$ by a separate path planning procedure for $q_{e}=q_{e}(s), s_{0} \leq s \leq s_{f}$, only. Hence, the trajectory planning/replanning is reduced then to the computation/adaptation of the velocity profile $\beta(\cdot)$ along a given fixed path $q_{e}(\cdot)=q_{e}^{(0)}(\cdot)$.

Assuming that the transformation $s=s(t)$ is differentiable on $\left[t_{0}, t_{f}\right]$ with the exception of at most a finite number of points, we introduce now the socalled velocity profile $\beta=\beta(s), s_{0} \leq s \leq s_{f}$, along the geometric path $\tilde{q}(\cdot)$ in configuration space by

$$
\beta(s):=\dot{s}^{2}(t(s))=\left(\frac{d s}{d t}\right)^{2}(t(s)),
$$


where $t=t(s), s_{0} \leq s \leq s_{f}$, is the inverse of $s=s(t), t_{0} \leq t \leq t_{f}$. Thus, we have that

$$
d t=\frac{1}{\sqrt{\beta(s)}} d s,
$$

and the time $t \geq t_{0}$ can be represented by the integral

$$
t=t(s):=t_{0}+\int_{s_{0}}^{s} \frac{d \sigma}{\sqrt{\beta(\sigma)}} .
$$

By using the integral transformation $\sigma:=s_{0}+\left(s-s_{0}\right) \rho, 0 \leq \rho \leq 1, t(s)$ may be represented also by

$$
t(s)=t_{0}+\left(s-s_{0}\right) \int_{0}^{1} \frac{d \rho}{\sqrt{\beta\left(s_{0}+\left(s-s_{0}\right) \rho\right)}}, s \geq s_{0} .
$$

By numerical quadrature, i.e., by applying a certain numerical integration formula of order $\nu$ and having weights $a_{1}, a_{2}, \ldots, a_{\nu}$ to the integral in (15a), the time function $t=t(s)$ can be represented approximatively by

$$
\tilde{t}(s):=t_{0}+\left(s-s_{0}\right) \sum_{k=0}^{\nu} \frac{a_{k}}{\sqrt{\beta\left(s_{0}+\left(s-s_{0}\right) \frac{k}{\nu}\right)}}, s \geq s_{0} .
$$

In case of Simpson's rule $(\nu=2)$ we have that

$$
\tilde{t}(s):=t_{0}+\frac{s-s_{0}}{6}\left(\frac{1}{\sqrt{\beta\left(s_{0}\right)}}+\frac{4}{\sqrt{\beta\left(\frac{s-s_{0}}{2}\right)}}+\frac{1}{\sqrt{\beta(s)}}\right) .
$$

As long as the basic mechanical equations, the cost and constraint functions do not depend explicitly on time $t$, the transformation of the robot control problem from the time onto the $s$-parameter domain causes no difficulties. In the more general case one has to use the time representation (14b), (15a) or its approximates $(15 b, c)$.

Obviously, the terminal time $t_{f}$ is given, cf. (14b), (15a), by

$$
\begin{aligned}
t_{f}=t\left(s_{f}\right) & =t_{0}+\int_{s_{0}}^{s_{f}} \frac{d \sigma}{\sqrt{\beta(\sigma)}} \\
& =t_{0}+\left(s_{f}-s_{0}\right) \int_{0}^{1} \frac{d \rho}{\sqrt{\beta\left(s_{0}+\left(s_{f}-s_{0}\right) \rho\right)}} .
\end{aligned}
$$




\subsection{Transformation of the dynamic equation}

Because of $(12 \mathrm{a}, \mathrm{b})$, we find

$$
\begin{aligned}
& \dot{q}(t)=q_{e}^{\prime}(s) \dot{s} \quad\left(\dot{s}:=\frac{d s}{d t}, q_{e}^{\prime}(s):=\frac{d \tilde{g}}{d s}\right) \\
& \ddot{q}(t)=q_{e}^{\prime}(s) \ddot{s}+q_{e}^{\prime \prime}(s) \dot{s}^{2} .
\end{aligned}
$$

Moreover, according to (13) we have that

$$
\dot{s}^{2}=\beta(s), \dot{s}=\sqrt{\beta(s)},
$$

and the differentiation of $(17 \mathrm{c})$ with respect to time $t$ yields

$$
\ddot{s}=\frac{1}{2} \beta^{\prime}(s) .
$$

Hence, (17a-d) yields the following representation

$$
\begin{aligned}
& \dot{q}(t)=q_{e}^{\prime}(s) \sqrt{\beta(s)} \\
& \ddot{q}(t)=q_{e}^{\prime}(s) \frac{1}{2} \beta^{\prime}(s)+q_{e}^{\prime \prime}(s) \beta(s)
\end{aligned}
$$

of $\dot{q}(t), \ddot{q}(t)$ in terms of the new unknown functions $q_{e}(\cdot), \beta(\cdot)$.

Inserting now $(18 \mathrm{a}, \mathrm{b})$ into the dynamic equation $(4 \mathrm{a})$, we find the equivalent relation

$$
u_{e}\left(p_{D}, s ; q_{e}(\cdot), \beta(\cdot)\right)=u(t) \text { with } s=s(t), t=t(s),
$$

where the function $u_{e}$ is defined by

$$
\begin{aligned}
u_{e}\left(p_{D}, s ; q_{e}(\cdot), \beta(\cdot)\right):= & M\left(p_{D}, q_{e}(s)\right)\left(\frac{1}{2} q_{e}^{\prime}(s) \beta^{\prime}(s)+q_{e}^{\prime \prime}(s) \beta(s)\right) \\
& +h\left(p_{D}, q_{e}(s), q_{e}^{\prime}(s) \sqrt{\beta(s)}\right) .
\end{aligned}
$$

The initial and terminal conditions (7a-c) are transformed, see (12a,b) and (18a), as follows

$$
\begin{gathered}
q_{e}\left(s_{0}\right)=\mathbf{q}_{0}(\omega), q_{e}^{\prime}\left(s_{0}\right) \sqrt{\beta\left(s_{o}\right)}=\dot{\mathbf{q}}_{\mathbf{0}}(\omega) \\
\psi\left(t\left(s_{f}\right), p, q_{e}\left(s_{f}\right), q_{e}^{\prime}\left(s_{f}\right) \sqrt{\beta\left(s_{f}\right)}\right)=0
\end{gathered}
$$

or

$$
q_{e}\left(s_{f}\right)=\mathbf{q}_{f}(\omega), q_{e}^{\prime}\left(s_{f}\right) \sqrt{\beta\left(s_{f}\right)}=\dot{\mathbf{q}}_{\mathbf{f}}(\omega)
$$

Remark 3.2. In most cases we have that the robot is at rest at time $t=t_{0}$ and $t=t_{f}$, i.e. $\dot{q}\left(t_{0}\right)=\dot{q}\left(t_{f}\right)=0$, hence, 


$$
\beta\left(s_{0}\right)=\beta\left(s_{f}\right)=0
$$

\subsection{Transformation of the control constraints}

Using $(12 \mathrm{a}, \mathrm{b})$, the control constraints (8a-c) read in $s$-form as follows:

$$
\begin{gathered}
u^{\min }\left(t(s), p_{C}\right) \leq u_{e}\left(p_{D}, s ; q_{e}(\cdot), \beta(\cdot)\right) \leq u^{\max }\left(t(s), p_{C}\right), s_{0} \leq s \leq s_{f}(21 \mathrm{a}) \\
g_{I}\left(t(s), p_{C}, q_{e}(s), q_{e}^{\prime}(s) \sqrt{\beta(s)}, u_{e}\left(p_{D}, s ; q_{e}(\cdot), \beta(\cdot)\right)\right) \leq 0, s_{0} \leq s \leq s_{f}(21 \mathrm{~b}) \\
g_{I I}\left(t(s), p_{C}, q_{e}(s), q_{e}^{\prime}(s) \sqrt{\beta(s)}, u_{e}\left(p_{D}, s ; q_{e}(\cdot), \beta(\cdot)\right)\right)=0, s_{0} \leq s \leq s_{f},(21 \mathrm{c})
\end{gathered}
$$

where $t=t(s)=t(s ; \beta(\cdot))$ or its approximation $t=\tilde{t}(s)=\tilde{t}(s ; \beta(\cdot))$ is defined by $(14 \mathrm{~b}),(15 \mathrm{a}-\mathrm{c})$.

Remark 3.3. 1. In the important case

$$
u^{\min }\left(t, p_{C}\right):=u^{\min }\left(p_{C}, q(t), \dot{q}(t)\right), u^{\max }\left(t, p_{C}\right):=u^{\max }\left(p_{C}, q(t), \dot{q}(t)\right)
$$

that the bounds for $u=u(t)$ depend on the system state $(q(t), \dot{q}(t))$ in configuration space, condition (21a) is reduced to

$$
\begin{array}{r}
u^{\min }\left(p_{C}, q_{e}(s), q_{e}^{\prime}(s) \sqrt{\beta(s)}\right) \leq u_{e}\left(p_{D}, s ; q_{e}(\cdot), \beta(\cdot)\right) \\
\leq u^{\max }\left(p_{C}, q_{e}(s), q_{e}^{\prime}(s) \sqrt{\beta(s)}\right), s_{0} \leq s \leq s_{f} .
\end{array}
$$

2. If the bounds for $u(t)$ in $(22 \mathrm{a})$ do not depend on the velocity $\dot{q}(t)$ in configuration space, and the geometric path $q_{e}(s)=q_{e}(s), s_{0} \leq s \leq s_{f}$, in configuration space is known in advance, then the bounds

$$
\begin{aligned}
& u^{\min }\left(p_{C}, q_{e}(s)\right)=\tilde{u}^{\min }\left(p_{C}, s\right) \\
& u^{\max }\left(p_{C}, q_{e}(s)\right)=\tilde{u}^{\max }\left(p_{C}, s\right), s_{0} \leq s \leq s_{f}
\end{aligned}
$$

depend on $\left(p_{C}, s\right)$ only.

Bounds of the type $(22 \mathrm{c})$ for the control function $u(t)$ may be taken into account as an approximation of the more general bounds in (21a).

\subsection{Transformation of the state constraints}

Applying the transformations $(12 \mathrm{a}, \mathrm{b}),(17 \mathrm{a})$ and $(14 \mathrm{~b})$ to the state constraints $(9 \mathrm{a}, \mathrm{b})$, we find the following $s$-form of the state constraints:

$$
S_{I}\left(t(s), p_{C}, q_{e}(s), q_{e}^{\prime}(s) \sqrt{\beta(s)}\right) \leq 0, s_{0} \leq s \leq s_{f}
$$




$$
S_{I I}\left(t(s), p_{C}, q_{e}(s), q_{e}^{\prime}(s) \sqrt{\beta(s)}\right)=0, s_{0} \leq s \leq s_{f}
$$

Obviously, the s-form of the special state constraints $(9 \mathrm{c}, \mathrm{d})$ read

$$
\begin{aligned}
& q_{\min }\left(p_{C}\right) \leq q_{e}(s) \leq q_{\max }\left(p_{C}\right), s_{0} \leq s \leq s_{f}, \\
& \dot{q}_{\min }\left(p_{C}\right) \leq q_{e}^{\prime}(s) \sqrt{\beta(s)} \leq \dot{q}_{\max }\left(p_{C}\right), s_{0} \leq s \leq s_{f} .
\end{aligned}
$$

In the case that the end-effector of the robot has to follow a given path (11) in work space, equation $(23 \mathrm{~b})$ reads

$$
T\left(p_{K}, q_{e}(s)\right)-x_{e}\left(p_{x}, s\right)=0, s_{0} \leq s \leq s_{f},
$$

where the parameter vector $p_{x}$ describes possible uncertainties in the selection of the path to be followed by the roboter in work space.

\subsection{Transformation of the objective formation}

Applying the integral transformation $t=t(s), d t=\frac{d s}{\sqrt{\beta(s)}}$ to the integral in the representation (6) of the objective function $J=J(u(\cdot))$, and transforming also the terminal costs, we find the following $s$-form of the objective function:

$$
\begin{gathered}
J(u(\cdot))=\int_{s_{0}}^{s_{f}} L\left(t(s), p_{J}, q_{e}(s), q_{e}^{\prime}(s) \sqrt{\beta(s)}, u_{e}\left(p_{D}, s ; q_{e}(\cdot), \beta(\cdot)\right)\right) \frac{d s}{\sqrt{\beta(s)}} \\
+\phi\left(t\left(s_{f}\right), p_{J}, q_{e}\left(s_{f}\right), q_{e}^{\prime}\left(s_{f}\right) \sqrt{\beta\left(s_{f}\right)}\right) .
\end{gathered}
$$

Note that $\beta\left(s_{f}\right)=0$ holds in many practical situations.

For the class of time-minimum problems we have that

$$
J(u(\cdot)):=t_{f}-t_{0}=\int_{t_{0}}^{t_{f}} d t=\int_{s_{0}}^{s_{f}} \frac{d s}{\sqrt{\beta(s)}} .
$$

\section{OSTP - Optimal Stochastic Trajectory Planning}

In the following we suppose that the initial and terminal conditions (20d) hold, i.e.

$$
\beta_{0}=\beta\left(s_{0}\right)=\beta_{f}=\beta\left(s_{f}\right)=0 \text { or } \dot{q}\left(t_{0}\right)=\dot{q}\left(t_{f}\right)=0 .
$$


Based on the $(t-s)$-transformation described in Section 3, and relying on the inverse dynamics approach, the robot control problem (6), (7a-c), (8a-c), (9ac) can be represented now by a variational problem for $\left(q_{e}(\cdot), \beta(\cdot)\right), \beta(\cdot)$, resp., given in the following. Having $\left(q_{e}(\cdot), \beta(\cdot)\right), \beta(\cdot)$, resp., a reference trajectory and a feedforward control can be constructed then.

\section{A) Time-invariant case (autonomous systems)}

If the objective function and the constraint functions do not depend explicitly on time $t$, then the optimal control problem takes the following equivalent $s$-forms:

$$
\min \int_{s_{0}}^{s_{f}} L^{J}\left(p_{J}, q_{e}(s), q_{e}^{\prime}(s), q_{e}^{\prime \prime}(s), \beta(s), \beta^{\prime}(s)\right) d s+\phi^{J}\left(p_{J}, q_{e}\left(s_{f}\right)\right)
$$

s.t.

$$
\begin{aligned}
& f_{I}^{u}\left(p, q_{e}(s), q_{e}^{\prime}(s), q_{e}^{\prime \prime}(s), \beta(s), \beta^{\prime}(s)\right) \leq 0, s_{0} \leq s \leq s_{f} \\
& f_{I I}^{u}\left(p, q_{e}(s), q_{e}^{\prime}(s), q_{e}^{\prime \prime}(s), \beta(s), \beta^{\prime}(s)\right)=0, s_{0} \leq s \leq s_{f} \\
& f_{I}^{S}\left(p, q_{e}(s), q_{e}^{\prime}(s), \beta(s)\right) \leq 0, s_{0} \leq s \leq s_{f} \\
& f_{I I}^{S}\left(p, q_{e}(s), q_{e}^{\prime}(s), \beta(s)\right)=0, s_{0} \leq s \leq s_{f} \\
& \beta(s) \geq 0, s_{0} \leq s \leq s_{f} \\
& q_{e}\left(s_{0}\right)=\mathbf{q}_{0}(\omega), q_{e}^{\prime}\left(s_{0}\right) \sqrt{\beta\left(s_{0}\right)}=\dot{\mathbf{q}}_{\mathbf{0}}(\omega) \\
& q_{e}\left(s_{f}\right)=\mathbf{q}_{f}(\omega), \beta\left(s_{f}\right)=\beta_{f} .
\end{aligned}
$$

Under condition (20d), a more general version of the terminal condition (25h) reads, cf. (20b),

$$
\psi\left(p, q_{e}\left(s_{f}\right)\right)=0, \beta\left(s_{f}\right)=\beta_{f}:=0
$$

Here,

$$
\begin{aligned}
& L^{J}=L^{J}\left(p_{J}, q_{e}, q_{e}^{\prime}, q_{e}^{\prime \prime}, \beta, \beta^{\prime}\right), \phi^{J}=\phi^{J}\left(p_{J}, q_{e}\right) \\
& f_{I}^{u}=f_{I}^{u}\left(p, q_{e}, q_{e}^{\prime}, q_{e}^{\prime \prime}, \beta, \beta^{\prime}\right), f_{I I}^{u}=f_{I I}^{u}\left(p, q_{e}, q_{e}^{\prime}, q_{e}^{\prime \prime}, \beta, \beta^{\prime}\right) \\
& f_{I}^{S}=f_{I}^{S}\left(p, q_{e}, q_{e}^{\prime}, \beta\right), f_{I I}^{S}=f_{I I}^{S}\left(p, q_{e}, q_{e}^{\prime}, \beta\right)
\end{aligned}
$$


are the functions representing the $s$-form of the objective function (24a), the constraint functions in the control constraints (21a-c), and in the state constraints (23a-e), respectively. Define then $f^{u}$ and $f^{S}$ by

$$
f^{u}:=\left(\begin{array}{c}
f_{I}^{u} \\
f_{I I}^{u}
\end{array}\right), f^{S}:=\left(\begin{array}{c}
f_{I}^{S} \\
f_{I I}^{S}
\end{array}\right) .
$$

B) Time-varying case (nonautonomous systems)

If the time $t$ occurs explicitly in the objective and/or in some of the constraints of the robot control problem, then, using (14a,b), (15a-c), we have that $t=t\left(s ; t_{0}, s_{0}, \beta(\cdot)\right)$, and the functions $(26 \mathrm{a}-\mathrm{d})$ and $\psi$ may depend then also on $\left(s, t_{0}, s_{0}, \beta(\cdot)\right),\left(s_{f}, t_{0}, s_{0}, \beta(\cdot)\right)$, resp., hence,

$$
\begin{aligned}
& L^{J}=L^{J}\left(s, t_{0}, s_{0}, \beta(\cdot), p_{J}, q_{e}, q_{e}^{\prime}, q_{e}^{\prime \prime}, \beta, \beta^{\prime}\right), \phi^{J}=\phi^{J}\left(s_{f}, t_{0}, s_{0}, \beta(\cdot), p_{J}, q_{e}\right) \\
& f^{u}=f^{u}\left(s, t_{0}, s_{0}, \beta(\cdot), p, q_{e}, q_{e}^{\prime}, q_{e}^{\prime \prime}, \beta, \beta^{\prime}\right), f^{S}=f^{S}\left(s, t_{0}, s_{0}, \beta(\cdot), p, q_{e}, q_{e}^{\prime}, \beta\right) \\
& \psi=\psi\left(s_{f}, t_{0}, s_{0}, \beta(\cdot), p, q_{e}\right) .
\end{aligned}
$$

In order to get a reliable optimal geometric path $q_{e}^{*}=q_{e}^{*}(s)$ in configuration open and a reliable optimal velocity profile $\beta^{*}=\beta^{*}(s), s_{0} \leq s \leq s_{f}$, being robust with respect to random parameter variations of $p=p(\omega)$, the variational problem (25a-h) under stochastic uncertainty must be replaced by an appropriate deterministic substitute problem which is defined according to the following principles [22-25,31], cf. also [21].

Assume first that the a priori information about the robot and its environment up to time $t_{0}$ is described by means of a $\sigma$-algebra $\mathcal{A}_{t_{0}}$, and let then

$$
P_{p(\cdot)}^{(0)}=P_{p(\cdot)}\left(\cdot \mid \mathcal{A}_{t_{0}}\right)
$$

denote the a priori distribution of the random vector $p=p(\omega)$ given $\mathcal{A}_{t_{0}}$.

Depending on the decision theoretical point of view, different approaches are possible, e.g. reliability-based substitute problems, belonging essentially to one of the following two basic classes of substitute problems:

I) Risk-constrained minimum expected cost problems

II) Expected total cost-minimum problems. 
Substitute problems are based usually on certain scalar or vectorial loss or cost functions

$$
\gamma_{I}^{u}, \gamma_{I I}^{u}, \gamma_{I}^{S}, \gamma_{I I}^{S}, \gamma^{\psi}, \ldots
$$

evaluating the violation of the random constraints $(25 \mathrm{~b}, \mathrm{c}),(25 \mathrm{~d}, \mathrm{e}),\left(25 \mathrm{~h}^{\prime}\right)$, respectively.

In the following all expectations are conditional expectations with respect to the a priori distribution $P_{p(\cdot)}^{(0)}$ of the random parameter vector $p(\omega)$. Moreover, the following compositions are introduced:

$$
\begin{aligned}
& f_{\gamma}^{u}:=\left(\begin{array}{c}
\gamma_{I}^{u}\left(f_{I}^{u}\right) \\
\gamma_{I I}^{u}\left(f_{I I}^{u}\right)
\end{array}\right), f_{\gamma}^{S}:=\left(\begin{array}{c}
\gamma_{I}^{S}\left(f_{i}^{S}\right) \\
\gamma_{I I}^{S}\left(f_{I I}^{S}\right)
\end{array}\right) \\
& \psi_{\gamma}:=\gamma^{\psi}(\psi) .
\end{aligned}
$$

Now the two basic types of substitute problems are described.

\section{I) Risk-based minimum expected cost problems}

Minimizing the expected (primal) costs $E\left(J\left(u(\cdot) \mid \mathcal{A}_{t_{0}}\right)\right.$, and demanding that the risk, i.e. the expected costs arising from the violation of the constraints of the variations problem (25a-h) do not exceed given upper bounds, in the time-invariant case we find the following substitute problem:

$$
\begin{gathered}
\min \int_{s_{0}}^{s_{f}} E\left(L^{J}\left(p_{J}, q_{e}(s), q_{e}^{\prime}(s), q_{e}^{\prime \prime}(s), \beta(s), \beta^{\prime}(s)\right) \mid \mathcal{A}_{t_{0}}\right) d s \\
+E\left(\phi^{J}\left(p_{J}, q_{e}\left(s_{f}\right)\right) \mid \mathcal{A}_{t_{0}}\right)
\end{gathered}
$$

s.t.

$$
\begin{aligned}
& E\left(f_{\gamma}^{u}\left(p, q_{e}(s), q_{e}^{\prime}(s), q_{e}^{\prime \prime}(s), \beta(s), \beta^{\prime}(s)\right) \mid \mathcal{A}_{t_{0}}\right) \leq \Gamma^{u}, s_{0} \leq s \leq s_{f} \\
& E\left(f_{\gamma}^{S}\left(p, q_{e}(s), q_{e}^{\prime}(s), \beta(s)\right) \mid \mathcal{A}_{t_{0}}\right) \leq \Gamma^{S}, s_{0} \leq s \leq s_{f} \\
& \beta(s) \geq 0, s_{0} \leq s \leq s_{f} \\
& q_{e}\left(s_{0}\right)=\bar{q}_{0}, q_{e}^{\prime}\left(s_{0}\right) \sqrt{\beta\left(s_{0}\right)}=\bar{q}_{0} \\
& q_{e}\left(s_{f}\right)=\bar{q}_{f}\left(\text { if } \phi^{J}=0\right), \beta\left(s_{f}\right)=\beta_{f}
\end{aligned}
$$

and the more general terminal condition (25h') is replaced by

$$
\beta\left(s_{f}\right)=\beta_{f}:=0, E\left(\psi_{\gamma}\left(p, q_{e}\left(s_{f}\right)\right) \mid \mathcal{A}_{t_{0}}\right) \leq \Gamma_{\psi}
$$


Here,

$$
\Gamma^{u}=\Gamma^{u}(s), \Gamma^{S}=\Gamma^{S}(s), \Gamma_{\psi}=\Gamma_{\psi}(s)
$$

denote scalar or vectorial upper risk bounds which may depend on the path parameter $s \in\left[s_{0}, s_{f}\right]$. Furthermore, the initial, terminal values $q_{0}, \dot{q}_{0}, q_{f}$ in (29e,f) are determined according to one of the following relations:

(a)

$$
\bar{q}_{0}:=\hat{q}\left(t_{0}\right), \overline{\dot{q}}_{0}:=\hat{\dot{q}}\left(t_{0}\right), \bar{q}_{f}:=\hat{q}\left(t_{f}\right),
$$

where $(\hat{q}(t), \hat{\dot{q}}(t))$ denotes an estimate, observation, etc., of the state in configuration space at time $t$;

(b)

$$
\begin{aligned}
\bar{q}_{0} & :=E\left(\mathbf{q}_{0}(\omega) \mid \mathcal{A}_{t_{0}}\right), \overline{\dot{q}}_{0}:=E\left(\dot{\mathbf{q}}_{\mathbf{0}}(\omega) \mid \mathcal{A}_{t_{0}}\right), \\
\bar{q}_{f}={\overline{q_{f}}}^{(0)} & :=E\left(\mathbf{q}_{f}(\omega) \mid \mathcal{A}_{t_{0}}\right),
\end{aligned}
$$

where $\mathbf{q}_{0}(\omega), \dot{\mathbf{q}}_{0}(\omega)$ is a random initial position, and $\mathbf{q}_{f}(\omega)$ is a random terminal position.

Having corresponding information about initial, terminal values $x_{0}, \dot{x}_{0}, x_{f}$ in work space, related equations for $q_{0}, \dot{q}_{0}, q_{f}$ may be obtained by means of the kinematic equation (5).

\section{Remark 4.1. Average constraints}

Taking the average of the pointwise constraints $(29 \mathrm{~b}, \mathrm{c})$ with respect to the path parameter $s, s_{0} \leq s \leq s_{f}$, we get the simplified integrated constraints

$$
\begin{aligned}
& \int_{s_{0}}^{s_{f}} E\left(f_{\gamma}^{u}\left(p, q_{e},(s), q_{e}^{\prime}(s), q_{e}^{\prime \prime}(s), \beta(s), \beta^{\prime}(s)\right) \mid \mathcal{A}_{t_{0}}\right) d s \leq \tilde{\Gamma}^{u} \\
& \int_{s_{0}}^{s_{f}} E\left(f_{\gamma}^{S}\left(p, q_{e}(s), q_{e}^{\prime}(s), \beta(s)\right) \mid \mathcal{A}_{t_{0}}\right) d s \leq \tilde{\Gamma}^{S}
\end{aligned}
$$

\section{Remark 4.2. Generalized area of admissible motion}

In generalization of the admissible area of motion $[18,24,28]$ for path planning problems with a prescribed geometrical path $q_{e}(\cdot)=\bar{q}_{e}(\cdot)$ in configuration space, for point-to-point problems the constraints (29b-i) define for each 
path point $s, s_{0} \leq s \leq s_{f}$, a generalized admissible area of motion for the vector

$$
\chi(s):=\left(q_{e}(s), q_{e}^{\prime}(s), q_{e}^{\prime \prime}(s), \beta(s), \beta^{\prime}(s)\right), s_{0} \leq s \leq s_{f},
$$

including information about the magnitude $\left(\beta(s), \beta^{\prime}(s)\right)$ of the motion as well as information about the direction $\left(q_{e}(s), q_{e}^{\prime}(s), q_{e}^{\prime \prime}(s)\right)$ of the motion.

\section{Remark 4.3. Problems with Chance Constraints}

Substitute problems having chance constraints are obtained if the loss functions $\gamma^{u}, \gamma^{S}$ for evaluating the violation of the inequality constraints in (25ah,h') are 0-1-functions, cf. [24].

To give a characteristic example, we demand that the control, state constraints (21a), (23c), (23d), resp., have to be fulfilled at least with probability $\alpha_{u}, \alpha_{1}, \alpha_{\dot{q}}$, hence,

$$
\begin{gathered}
P\left(u^{\min }\left(p_{C}\right) \leq u_{e}\left(p_{D}, s ; q_{e}(\cdot), \beta(\cdot)\right) \leq u^{\max }\left(p_{C}\right) \mid \mathcal{A}_{t_{0}}\right) \geq \alpha_{u} \\
s_{0} \leq s \leq s_{f} \\
P\left(q^{\min }\left(p_{C}\right) \leq q_{e}(s) \leq q^{\max }\left(p_{C}\right) \mid \mathcal{A}_{t_{0}}\right) \geq \alpha_{q}, s_{0} \leq s \leq s_{f} \\
P\left(\dot{q}^{\min }\left(p_{C}\right) \leq q_{e}^{\prime}(s) \sqrt{\beta(s)} \leq q^{\max }\left(p_{C}\right) \mid \mathcal{A}_{t_{0}}\right) \geq \alpha_{\dot{q}}, s_{0} \leq s \leq s_{f} .
\end{gathered}
$$

Sufficient conditions for the complicated chance constraints (30a-c) can be obtained by applying certain probability inequalities, see [24]. Defining

$$
u^{c}\left(p_{C}\right):=\frac{u^{\max }\left(p_{C}\right)+u^{\min }\left(p_{C}\right)}{2}, \rho_{u}\left(p_{C}\right):=\frac{u^{\max }\left(p_{C}\right)-u^{\min }\left(p_{C}\right)}{2},
$$

then a sufficient conditions for (30a) reads, cf. [24],

$$
\begin{array}{r}
E\left(\operatorname{trB} \rho_{u}\left(p_{C}\right)_{d}^{-1}\left(u_{e}-u^{c}\left(p_{C}\right)\right)\left(u_{e}-u^{c}\left(p_{C}\right)\right)^{\prime} \rho_{u}\left(p_{C}\right)_{d}^{-1} \mid \mathcal{A}_{t_{0}}\right) \\
\leq 1-\alpha_{u}, s_{o} \leq s \leq s_{f}
\end{array}
$$

where $u_{e}=u_{e}\left(p_{D}, s ; q_{e}(\cdot), \beta(\cdot)\right)$ and $\rho_{u}\left(p_{C}\right)_{d}$ denotes the diagonal matrix containing the elements of $\rho_{u}\left(p_{C}\right)$ on its diagonal. Moreover, $B$ denotes a positive definite matrix such that $z^{\prime} B z \geq 1$ for all vectors $z$ such that $\|z\|_{\infty} \geq 1$. Taking e.g. $B=I,(30 \mathrm{e})$ reads

$$
\begin{array}{r}
E\left(\left\|\rho_{u}\left(p_{C}\right)_{d}^{-1}\left(u_{e}\left(p_{D}, s ; q_{e}(\cdot), \beta(\cdot)\right)-u^{c}\left(p_{C}\right)\right)\right\|^{2} \mid \mathcal{A}_{t_{0}}\right) \leq 1-\alpha_{u} \\
s_{0} \leq s \leq s_{f} .
\end{array}
$$


Obviously, similar sufficient conditions may be derived for (30b,c).

We observe that the above class of risk-based minimum expected cost problems for the computation of $\left(q_{e}(\cdot), \beta(\cdot)\right), \beta(\cdot)$, resp., is represented completely by the following set of

$$
\text { initial parameters } \zeta_{0}: t_{0}, s_{0}, \bar{q}_{0}, \overline{\dot{q}}_{0}, P_{p(\cdot)}^{(0)} \text { or } \nu_{0}
$$

and

$$
\text { terminal parameters } \zeta_{f}: t_{f}, s_{f}, \beta_{f}, \bar{q}_{f},
$$

where in case of problems with a given geometric path $q_{e}=q_{e}(s)$ in configuration space, the values $q_{0}, q_{f}$ may be deleted. Moreover, approximating the expectations in (29a-f,f') by means of Taylor expansions with respect to the parameter vector $p$ at the conditional mean

$$
\bar{p}^{(0)}:=E\left(p(\omega) \mid \mathcal{A}_{t_{0}}\right),
$$

the a priori distribution $P_{p(\cdot)}^{(0)}$ may be replaced by a certain vector

$$
\nu_{0}:=\left(E\left(\prod_{k=1}^{r} p_{l}(\omega) \mid \mathcal{A}_{t_{0}}\right)_{\left(l_{1}, \ldots, l_{r}\right) \in \Lambda}\right)
$$

of a priori moments of $p(\omega)$ with respect to $\mathcal{A}_{t_{0}}$.

Here, $\Lambda$ denotes a certain finite set of multiple indices $\left(l_{1}, \ldots, l_{\tau}\right), r \leq 1$.

In the time-variant case, the functions $f^{J}, \phi^{J}$ are given by (26a'), and the functions $f_{\gamma}^{u}, g_{\gamma}^{u}, f_{\gamma}^{S}, g_{\gamma}^{S}, \psi_{\gamma}$ are defined again by (28b-d), where $f^{u}, g^{u}, f^{S}, g^{S}, \psi$ are given now by (26b'-d'). Obviously $t_{0}, t_{f}$ occurs now explicitly in the parameter list $(31 \mathrm{a}, \mathrm{b})$.

II) Expected total cost-minimum problem

Here, the total costs, cf. (29b',c'), arising from violations of the constraints in the variational problem (29a-f,f') are added - after a certain weighting which may be included of course in the cost or loss functions (28a) - to the (primary) costs arising along the trajectory and to the terminal costs.

Taking the expectations with respect to $\mathcal{A}_{t_{0}}$, in the time-invariant case the following substitute problem is obtained:

$$
\min \int_{s_{0}}^{s_{f}} E\left(L_{\gamma}^{J}\left(p, q_{e}(s), q_{e}^{\prime}(s), q_{e}^{\prime \prime}(s), \beta(s), \beta^{\prime}(s)\right) \mid \mathcal{A}_{t_{0}}\right) d s
$$




$$
+E\left(\phi_{\gamma}^{J}\left(p, q_{e}\left(s_{f}\right)\right) \mid \mathcal{A}_{t_{0}}\right)
$$

s.t.

$$
\begin{aligned}
\beta(s) & \geq 0, s_{0} \leq s \leq s_{f} \\
q_{e}\left(s_{0}\right) & =\bar{q}_{0}, q_{e}^{\prime}\left(s_{0}\right) \sqrt{\beta\left(s_{0}\right)}=\overline{\dot{q}}_{0} \\
q_{e}\left(s_{f}\right) & =\bar{q}_{f}\left(\text { if } \phi_{\gamma}^{J}=0\right), \beta\left(s_{f}\right)=\beta_{f} .
\end{aligned}
$$

where $L_{\gamma}^{J}, \phi_{\gamma}^{J}$ are defined by

$$
\begin{aligned}
& L_{\gamma}^{J}:=L^{J}+\lambda_{I}^{u T} f_{I}^{u}+\lambda_{I I}^{u T} f_{I I}^{u}+\lambda_{I}^{S T} f_{I}^{S}+\lambda_{I I}^{S T} g_{I I}^{S} \\
& \phi_{\gamma}^{J}:=\phi^{J} \text { or } \phi_{\gamma}^{J}:=\phi^{J}+\lambda_{\psi}^{T} \psi_{\gamma}
\end{aligned}
$$

and $\lambda_{I}^{u}, \lambda_{I I}^{u}, \lambda_{I}^{S}, \lambda_{I I}^{S}, \lambda_{\psi} \geq 0$ are certain nonnegative (vectorial) scale factors which may depend on the path parameter $s$.

We observe that also in this case the initial/terminal parameters characterizing the second class of substitute problems (32a-f) are given again by $(31 \mathrm{a}, \mathrm{b})$.

In the time-varying case the present class (II) of substitute problems reads, cf. $\left(26 \mathrm{a}^{\prime}-\mathrm{c}^{\prime}\right)$ :

$$
\begin{array}{r}
\min \int_{s_{0}}^{s_{f}} E\left(L_{\gamma}^{J}\left(s, t_{0}, s_{0}, \beta(\cdot), p_{J}, q_{e}(s), q_{e}^{\prime}\left(s, q_{e}^{\prime \prime}(s), \beta(s), \beta^{\prime}(s)\right) \mid \mathcal{A}_{t_{0}}\right) d s\right. \\
+E\left(\phi_{\gamma}^{J}\left(s_{f}, t_{0}, s_{0}, \beta(\cdot), p_{J}, q_{e}\left(s_{f}\right)\right) \mid \mathcal{A}_{t_{0}}\right)
\end{array}
$$

s.t.

$$
\begin{gathered}
\beta(s) \geq 0, s_{0} \leq s \leq s_{f} \\
q_{e}\left(s_{0}\right)=q_{0}, q_{e}^{\prime}\left(s_{0}\right) \sqrt{\beta\left(s_{0}\right)}=\dot{q}_{0} \\
q_{e}\left(s_{f}\right)=q_{f}\left(\text { if } \phi_{\gamma}^{J}=0\right), \beta\left(s_{f}\right)=\beta_{f} .
\end{gathered}
$$

Remark 4.4. Mixtures of (I), (II)

Several mixtures of the classes (I) and (II) of substitute problems are possible.

\subsection{Computational aspects}

The following techniques are available for solving substitutes problems of type (I), (II): 
a) Reduction to a finite dimensional parameter optimization problem

Here, the unknown functions $\left(q_{e}(\cdot), \beta(\cdot)\right)$ or $\beta(\cdot)$ are approximated by a linear combination

$$
\begin{aligned}
q_{e}(s) & :=\sum_{l=1}^{l_{q}} \hat{q}_{l} B_{l}(s), s_{0} \leq s \leq s_{f} \\
\beta(s) & :=\sum_{l=1}^{l_{\beta}} \hat{\beta}_{l} B_{l}^{\beta}(s), s_{0} \leq s \leq s_{f},
\end{aligned}
$$

where $B_{l}=B_{l}(s), B_{l}^{\beta}=B_{l}^{\beta}(s), s_{0} \leq s \leq s_{f}, l=1, \ldots, l_{q}\left(l_{\beta}\right)$, are given basis functions, e.g. B-splines, and $\hat{q}_{l}, \hat{\beta}_{l}, l=1, \ldots, l_{q}\left(l_{\beta}\right)$, are vectorial, scalar coefficients. Putting (34a,b) into (29a-f,f'), (32a-c), resp., a semiinfinite optimization problem is obtained. If the inequalities involving explicitly the path parameter $s, s_{0} \leq s \leq s_{f}$, are required for a finite number $N$ of parameter values $s_{1}, s_{2}, \ldots, s_{n}$ only, then this problem is reduced finally to a finite dimensional parameter optimization problem which can be solved now numerically by standard mathematical programming routines or search techniques. Of course, a major problem is the approximative computation of the conditional expectations which is done essentially by means of Taylor expansion with respect to the parameter vector $p$ at $\bar{p}^{(0)}$. Consequently, several conditional moments have to be determined (on-line, for stage $j \geq 1$ ). For details, see $[22,23,25,31]$ and the program package "OSTP" [3].

b) Variational techniques

Using methods from calculus of variations, necessary and - in some cases also sufficient conditions in terms of certain differential equations may be derived for the optimal solutions $\left(q_{e}^{(0)}, \beta^{(0)}\right), \beta^{(0)}$, resp., of the variational problems (29a-f,f'), (32a-c). For more details, see [31].

\section{c) Linearization methods}

Here, we assume that we already have an approximative optimal solution $\left(\bar{q}_{e}(s), \bar{\beta}(s)\right), s_{0} \leq s \leq s_{f}$, of the substitute problem (29a-f,f') or (32a-f) under consideration. E.g., an approximative optimal solution $\left(\bar{q}_{e}(\cdot), \bar{\beta}(\cdot)\right)$ can be obtained by starting from the deterministic substitute problem obtained by replacing the random parameter vector $p(\omega)$ just by its conditional mean $\bar{p}^{(0)}:=E\left(p(\omega) \mid \mathcal{A}_{t_{0}}\right)$. 
Given an approximate optimal solution $\left(\bar{q}_{e}(\cdot), \bar{\beta}(\cdot)\right)$ of substitute problem (I) or (II), the unknown optimal solution $\left(q_{e}^{(0)}(\cdot), \beta^{(0)}(\cdot)\right)$ to be determined is represented by

$$
\begin{aligned}
& q_{e}^{(0)}(s):=\bar{q}_{e}(s)+\Delta q_{e}(s), s_{0} \leq s \leq s_{f} \\
& \beta^{(0)}(s):=\bar{\beta}(s)+\Delta \beta(s), s_{0} \leq s \leq s_{f}
\end{aligned}
$$

where $\left(\Delta q_{e}(s), \Delta \beta(s)\right), s_{0} \leq s \leq s_{f}$, are certain (small) correction terms. In the following we assume that the changes $\Delta q_{e}(s), \Delta \beta(s)$ and their first and resp. second order derivatives $\Delta q_{e}^{\prime}(s), \Delta q_{e}^{\prime \prime}(s), \Delta \beta^{\prime}(s)$ are small.

We observe that the function arising in the constraints and in the objective of (29a-f,f'), (32a-f), resp., are of the following type:

$$
\begin{aligned}
\bar{g}^{(0)}\left(q_{e}(s), q_{e}^{\prime}(s), q_{e}^{\prime \prime}(s), \beta(s), \beta^{\prime}(s)\right):= & E\left(g \left(p(\omega), q_{e}(s), q_{e}^{\prime}(s),\right.\right. \\
& \left.\left.q_{e}^{\prime \prime}(s), \beta(s), \beta^{\prime}(s)\right) \mid \mathcal{A}_{t_{0}}\right), \\
\bar{\phi}^{(0)}\left(q_{e}\left(s_{f}\right)\right):=E\left(\phi\left(p(\omega), q_{e}\left(s_{f}\right)\right) \mid \mathcal{A}_{t_{0}}\right) &
\end{aligned}
$$

and

$$
\bar{F}^{(0)}\left(q_{e}(\cdot), \beta(\cdot)\right):=\int_{s_{0}}^{s_{f}} \bar{g}^{(0)}\left(q_{e}(s), q_{e}^{\prime}(s), q_{e}^{\prime \prime}(s), \beta(s), \beta^{\prime}(s)\right) d s .
$$

with certain functions $g, \phi$. Moreover, if for simplification the pointwise (cost-) constraints $(29 \mathrm{~b}, \mathrm{c})$ are averaged with respect to the path parameter $s, s_{0} \leq s_{f}$, then also constraint functions of the type (36c) arise, see (29b',c'). By means of first order Taylor expansion of $g, \phi$ with respect to $\left(\Delta q_{e}(s), \Delta q_{e}^{\prime}(s), \Delta q_{e}^{\prime \prime}(s), \Delta \beta(s), \Delta \beta^{\prime}(s)\right)$ at $\left(\bar{q}_{e}(s), \bar{q}_{e}^{\prime}(s), \bar{q}_{e}^{\prime \prime}(s), \bar{\beta}(s), \bar{\beta}^{\prime}(s)\right), s_{0} \leq$ $s \leq s_{f}$, we find then the following approximations

$$
\begin{aligned}
& \bar{g}^{(0)}\left(q_{e}(s), q_{e}^{\prime}(s), q_{e}^{\prime \prime}(s), \beta(s), \beta^{\prime}(s)\right) \\
& \approx \bar{g}^{(0)}\left(\bar{q}_{e}(s), \bar{q}_{e}^{\prime}(s), \bar{q}_{e}^{\prime \prime}(s), \bar{\beta}(s), \bar{\beta}^{\prime}(s)\right) \\
& +\bar{A}_{g, \bar{q}_{e}, \bar{\beta}}^{(0)}(s)^{T} \Delta q_{e}(s)+\bar{B}_{g, \bar{q}_{e}, \bar{\beta}^{(s)}}^{(0)} \Delta q_{e}^{\prime}(s)+\bar{C}_{g, \bar{q}_{e}, \bar{\beta}}^{(0)}(s)^{T} \Delta q_{e}^{\prime \prime}(s)
\end{aligned}
$$

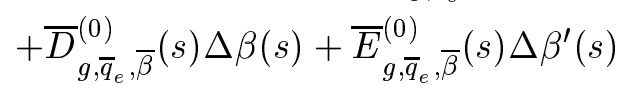

and

$$
\bar{\phi}^{(0)}\left(q_{e}\left(s_{f}\right)\right) \approx \quad \bar{\phi}^{(0)}\left(\bar{q}_{e}\left(s_{f}\right)\right) \quad+\bar{a}_{\phi, \bar{q}_{e}}^{(0)}\left(s_{f}\right)^{T} \Delta q_{e}\left(s_{f}\right),
$$


where the expected sensitivities of $g, \phi$ with respect to $q, q^{\prime}, q^{\prime \prime}, \beta$ and $\beta^{\prime}$ are given by

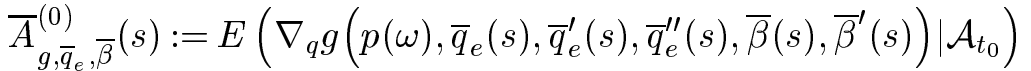

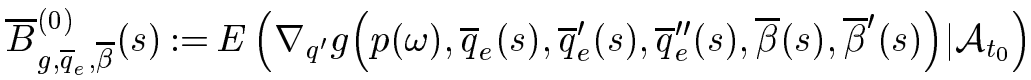

$$
\begin{aligned}
& \bar{C}_{g, \bar{q}_{e}, \bar{\beta}}^{(0)}(s):=E\left(\nabla_{q^{\prime \prime}} g\left(p(\omega), \bar{q}_{e}(s), \bar{q}_{e}^{\prime}(s), \bar{q}_{e}^{\prime \prime}(s), \bar{\beta}(s), \bar{\beta}^{\prime}(s)\right) \mid \mathcal{A}_{t_{0}}\right) \\
& \bar{D}_{g, \bar{q}_{e}, \bar{\beta}}^{(0)}(s):=E\left(\frac{\partial g}{\partial \beta}\left(p(\omega), \bar{q}_{e}(s), \bar{q}_{e}^{\prime}(s), \bar{q}_{e}^{\prime \prime}(s), \bar{\beta}(s), \bar{\beta}^{\prime}(s)\right) \mid \mathcal{A}_{t_{0}}\right)
\end{aligned}
$$

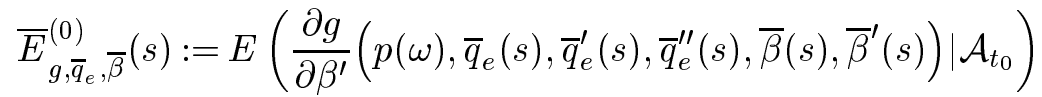

$$
\begin{aligned}
& \bar{a}_{\phi, \bar{q}_{e}}^{(0)}\left(s_{f}\right):=E\left(\nabla_{q} \phi\left(p(\omega), \bar{q}_{e}(s)\right) \mid \mathcal{A}_{t_{0}}\right), s_{0} \leq s \leq s_{\phi} .
\end{aligned}
$$

As mentioned before, cf. (31c,d), the expected values $\bar{g}^{(0)}, \bar{\phi}^{(0)}$ in $(37 \mathrm{a}, \mathrm{b})$ and the expected sensitivities defined by $(37 \mathrm{c}-\mathrm{h})$ can be computed approximatively by means of Taylor expansion with respect to $p$ at $\bar{p}^{(0)}=E\left(p(\omega) \mid \mathcal{A}_{t_{0}}\right)$. According to (37a), and using partial integration, for the total costs $\bar{F}^{(0)}$ along the path we get the following approximation:

$$
\begin{aligned}
& \bar{F}^{(0)} \approx \int_{s_{0}}^{s_{f}} \bar{g}^{(0)}\left(\bar{q}_{e}(s), \bar{q}_{e}^{\prime}(s), \bar{q}_{e}^{\prime \prime}(s), \bar{\beta}(s), \bar{\beta}^{\prime}(s)\right) d s \\
& +\int_{s_{0}}^{s_{f}} \bar{G}_{g, \bar{q}_{e}, \bar{\beta}}^{(0)}(s)^{T} \Delta q_{e}(s) d s+\int_{s_{0}}^{s_{f}} \bar{H}_{g, \bar{q}_{e}, \bar{\beta}}^{(0)}(s) \Delta \beta(s) d s
\end{aligned}
$$

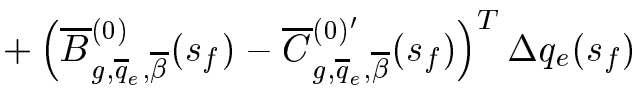

$$
\begin{aligned}
& +\left(-\bar{B}_{g, \bar{q}_{e}, \bar{\beta}}^{(0)}\left(s_{0}\right)+\bar{C}_{g, \bar{q}_{e}, \bar{\beta}^{\prime}}^{(0)^{\prime}}\left(s_{0}\right)\right)^{T} \Delta q_{e}\left(s_{0}\right)
\end{aligned}
$$

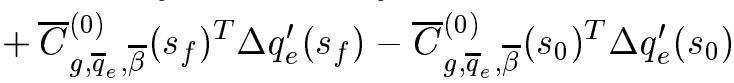

$$
\begin{aligned}
& +\bar{E}_{g, \bar{q}_{e}, \bar{\beta}}^{(0)}\left(s_{f}\right) \Delta \beta\left(s_{f}\right)-\bar{E}_{g, \bar{q}_{e}, \bar{\beta}}^{(0)}\left(s_{0}\right) \Delta \beta\left(s_{0}\right),
\end{aligned}
$$

where

$$
\begin{aligned}
\bar{G}_{g, \bar{q}_{e}, \bar{\beta}}^{(0)}(s) & :=\bar{A}_{g, \bar{q}_{e}, \bar{\beta}}^{(0)}(s)-\bar{B}_{g, \bar{q}_{e}, \bar{\beta}^{\prime}}^{(0)^{\prime}}(s)+\bar{C}_{g, \bar{q}_{e}, \bar{\beta}}^{(0)^{\prime \prime}}(s) \\
\bar{H}_{g, \bar{q}_{e}, \bar{\beta}}^{(0)}(s) & :=\bar{D}_{g, \bar{q}_{e}, \bar{\beta}}^{(0)}(s)-\bar{E}_{g, \bar{q}_{e}, \bar{\beta}}^{(0)^{\prime}}(s) .
\end{aligned}
$$

Conditions (29e-f,f'), (32c,d), resp., yield the following initial and terminal conditions for the changes $\Delta q_{e}(s), \Delta \beta(s)$ :

$$
\begin{aligned}
& \Delta \beta\left(s_{0}\right)=0, \Delta q_{e}\left(s_{0}\right)=\bar{q}_{0}-\bar{q}_{e}\left(s_{0}\right) \\
& \Delta \beta\left(s_{f}\right)=0, \Delta q_{e}\left(s_{f}\right)=\bar{q}_{f}-\bar{q}_{e}\left(s_{f}\right), \text { if } \phi^{J}=0 .
\end{aligned}
$$


Moreover, if $\dot{q}_{0} \neq 0$ (as in later correction stages, cf. Section 5), according to (29e) or (32c), condition $\Delta \beta\left(s_{0}\right)=0$ must be replaced by the more general one

$$
\left(\bar{q}_{e}^{\prime}\left(s_{0}\right)+\Delta q_{e}^{\prime}\left(s_{0}\right)\right) \sqrt{\bar{\beta}\left(s_{0}\right)+\Delta \beta\left(s_{0}\right)}=\bar{q}_{0}
$$

which can be approximated by

$$
\sqrt{\bar{\beta}\left(s_{0}\right)} \Delta q_{e}^{\prime}\left(s_{0}\right)+\frac{1}{2} \frac{\Delta \beta\left(s_{0}\right)}{\sqrt{\bar{\beta}\left(s_{0}\right)}} \bar{q}_{e}^{\prime}\left(s_{0}\right) \approx \bar{q}_{0}-\sqrt{\bar{\beta}\left(s_{0}\right)} \bar{q}_{e}^{\prime}\left(s_{0}\right) .
$$

Applying the above described linearization (37a-h) to (29a-e) or to the constraints $(29 \mathrm{~b}, \mathrm{c})$ only, problem $\left(29 \mathrm{a}-\mathrm{f}, \mathrm{f}^{\prime}\right)$ is approximated by a linear variational problem or a variational problem having linear constraints for the changes $\Delta q_{e}(\cdot), \Delta \beta(\cdot)$. On the other hand, using linearizations of the type (37a-h) in the variational problem (32a-f), in the average constraints (29b',c'), resp., an optimization problem for $\Delta q_{e}(\cdot), \Delta \beta(\cdot)$ is obtained which is linear, which has linear constraints, respectively.

d) Separated computation of $q_{e}(\cdot)$ and $\beta(\cdot)$

In order to reduce the computational complexity, the given trajectory planning problem is often split up [15] into the following two separated problems for $q_{e}(\cdot)$ and $\beta(\cdot)$ :

i) Optimal path planning: find the shortest collision-free geometric path $q_{e}^{(0)}=q_{e}^{(0)}(s), s_{0} \leq s \leq s_{f}$, in configuration space from a given initial point $q_{0}$ to a prescribed terminal point $q_{f}$. Alternatively, with a given initial velocity profile $\beta(\cdot)=\bar{\beta}(\cdot)$, see (35b), the substitute problem (29af,f'), (32a-f), resp., may be solved for an approximate geometric path $q_{e}(\cdot)=q_{e}^{(0)}(\cdot)$ only.

ii) Velocity planning: Determine then an optimal velocity profile $\beta^{(0)}=$ $\beta^{(0)}(s), s_{0} \leq s \leq s_{f}$, along the predetermined path $q_{e}^{(0)}(\cdot)$.

Having a certain collection $\left\{q_{e}, \lambda(\cdot): \lambda \in \Lambda\right\}$ of admissible paths in configuration space, a variant of the above procedure (i), (ii) is to determine - in an inner optimization loop - the optimal velocity profile $\beta_{\lambda}(\cdot)$ with respect to a given path $q_{e, \lambda}(\cdot)$, and to optimize then the parameter $\lambda$ in an outer optimization loop, see [18]. 


\subsection{Optimal reference trajectory, optimal feedforward control}

Having, at least approximatively, the optimal geometric path $q_{e}^{(0)}=q_{e}^{(0)}(s)$ and the optimal velocity profile $\beta^{(0)}=\beta^{(0)}(s), s_{0} \leq s \leq s_{f}$, i.e. the optimal solution $\left(q_{e}^{(0)}, \beta^{(0)}\right)=\left(q_{e}^{(0)}(s), \beta^{(0)}(s)\right), s_{0} \leq s \leq s_{f}$, of one of the stochastic path planning problems (29a-f,f'), (32a-f), (33a-d), resp., then, according to (12a,b), (13), the optimal reference trajectory in configuration space $q^{(0)}=q^{(0)}(t), t \geq t_{0}$, is defined by

$$
q^{(0)}(t):=q_{e}^{(0)}\left(s^{(0)}(t)\right), t \geq t_{0},
$$

where the optimal $(t \leftrightarrow s)$-transformation $s^{(0)}=s^{(0)}(t), t \geq t_{0}$, is defined by the initial values problem

$$
\dot{s}(t)=\sqrt{\beta^{(0)}(s)}, t \geq t_{0}, s^{(0)}(t):=s_{0} .
$$

By means of the kinematic equation (5), the corresponding reference trajectory $x^{(0)}=x^{(0)}(t), t \geq t_{0}$, in workspace may be defined by

$$
x^{(0)}(t):=E\left(T\left(p_{K}(\omega), q^{(0)}(t)\right) \mid \mathcal{A}_{t_{0}}\right)=T\left(\bar{p}_{K}^{(0)}, q^{(0)}(t)\right), t \geq t_{0},
$$

where

$$
\bar{p}_{K}^{(0)}:=E\left(p_{K}(\omega) \mid \mathcal{A}_{t_{0}}\right)
$$

Based on the inverse dynamics approach, see Remark 2.1, the optimal reference trajectory $q^{(0)}=q^{(0)}(t), t \geq t_{0}$, is inserted now into the left hand side of the dynamic equation (4a). This yields next to the random optimal control function

$$
\begin{aligned}
v^{(0)}\left(t, p_{D}(\omega)\right): & =M\left(p_{D}(\omega), q^{(0)}(t)\right) \ddot{q}^{(0)}(t) \\
& +h\left(p_{D}(\omega), q^{(0)}(t), \dot{q}^{(0)}(t)\right), t \geq t_{0} .
\end{aligned}
$$

Starting at the initial state $\left(q_{0}, \dot{q}_{0}\right):=\left(q^{(0)}\left(t_{0}\right), \dot{q}^{(0)}\left(t_{0}\right)\right)$, this control obviously keeps the robot exactly on the optimal trajectory $q^{(0)}(t), t \geq t_{0}$, provided that $p_{D}(\omega)$ is the true vector of dynamic parameters.

An optimal feedforward control law $u^{(0)}=u^{(0)}(t), t \geq t_{0}$, related to the optimal reference trajectory $q^{(0)}=q^{(0)}(t), t \geq t_{0}$, can be obtained then by applying a certain averaging or estimating operator $\Psi=\Psi\left(\cdot \mid \mathcal{A}_{t_{0}}\right) t_{0}(40)$ :

$$
u^{(0)}:=\Psi\left(v^{(0)}\left(t, p_{D}(\cdot)\right) \mid \mathcal{A}_{t_{0}}\right), t \geq t_{0} .
$$


If $\Psi\left(\cdot \mid \mathcal{A}_{t_{0}}\right)$ is the conditional expectation, then we find the optimal feedforward control law

$$
\begin{aligned}
u^{(0)} & :=E\left(M\left(p_{D}(\omega), q^{(0)}(t)\right) \ddot{q}^{(0)}(t)+h\left(p_{D}(\omega), q^{(0)}(t), \dot{q}^{(0)}(t)\right) \mid \mathcal{A}_{t_{0}}\right), \\
& =M\left(\bar{p}_{D}^{(0)}, q^{(0)}(t)\right) \ddot{q}^{(0)}(t)+h\left(\bar{p}_{D}^{(0)}, q^{(0)}(t), \dot{q}^{(0)}(t)\right), t \geq t_{0},
\end{aligned}
$$

where $\bar{p}_{D}^{(0)}$ denotes the conditional mean of $p_{D}(\omega)$ defined by (31c), and the second equation in formula (42a) holds since the dynamic equation of a robot depends linearly on the parameter vector $p_{D}$, see Remark 2.2 .

Inserting into the dynamic equation (4a), instead of the conditional mean $\bar{p}_{D}^{(0)}$ of $p_{D}(\omega)$ given $\mathcal{A}_{t_{0}}$, another estimator $p_{D}^{(0)}$ of the true parameter vector $p_{D}$ or a certain realization $p_{D}^{(0)}$ of $p_{D}(\omega)$ at the time instant $t_{0}$, then we obtain the optimal feedforward control law

$$
u^{(0)}(t):=M\left(p_{D}^{(0)}, q^{(0)}(t)\right) \ddot{q}^{(0)}(t)+h\left(p_{D}^{(0)}, q^{(0)}(t), \dot{q}^{(0)}(t)\right), t \geq t_{0} .
$$

\section{AOSTP - Adaptive Optimal Stochastic Trajectory Planning}

As mentioned already in the introduction, by means of direct or indirect measurements, observations of the robot and its environment, as e.g. by observations of the state $(x, \dot{x}),(q, \dot{q})$, resp., of the mechanical system in work or configuration space, further informations about the unknown parameter vector $p=p(\omega)$ are available at each time instant $t>t_{0}$. Let denote, cf. Sections 1,4 ,

$$
\mathcal{A}_{t}(\subset \mathcal{A}), t \geq t_{0}
$$

the $\sigma$-algebra of all information about the random parameter vector $p=p(\omega)$ up to time $t$.

By parameter identification $[17,36]$ or robot calibration techniques $[5,37]$ we may determine then the conditional distribution

$$
P_{p(\cdot)}^{(t)}=P_{p(\cdot) \mid \mathcal{A}_{t}}
$$

of $p(\omega)$ given $\mathcal{A}_{t}$, or we may determine the vector of conditional moments

$$
\nu^{(t)}:=\left(E\left(\prod_{k=1}^{r} p_{l_{k}}(\omega) \mid \mathcal{A}_{t}\right)\right)_{\left(l_{1}, \ldots, l_{r}\right) \in \Lambda}
$$


arising in the approximate computation of conditional expectations in (OSTP) with respect to $\mathcal{A}_{t}$, cf. $(31 \mathrm{c}, \mathrm{d})$.

The increase of information about the unknown parameter vector $p(\omega)$ from one time instant $t$ to the next $t+d t$ may be rather low, and the determination of $P_{p(\cdot)}^{(t)}$ or $\nu_{t}$ at each time point $t$ be very expensive, though identification methods in realtime exist [36]. Hence, as already mentioned briefly in Section 1, the conditional distribution $P_{p(\cdot)}^{(t)}$ or the vector of conditional moments $\nu_{t}$ is determined/updated at discrete time instants $\left(t_{j}\right)$ :

$$
t_{0}<t_{1}<t_{2}<\ldots<t_{j}<t_{j+1}<\ldots
$$

The optimal functions $q_{e}^{(0)}(s), \beta^{(0)}(s), s_{0} \leq s \leq s_{f}$, based on the a priori information $\mathcal{A}_{t_{0}}$, lose in course of time more or less their qualification to provide a satisfactory pair of guiding functions $\left(q^{(0)}(t), u^{(0)}(t)\right), t \geq t_{0}$.

However, having at the main correction time points $t_{j}, j=1,2, \ldots$, the updated information $\sigma$-algebras $\mathcal{A}_{t_{j}}$ and then the a posteriori probability distributions $P_{p(\cdot)}^{\left(t_{j}\right)}$ or the updated conditional moments $\nu^{\left(t_{j}\right)}$ of $p(\omega), j=1,2, \ldots$, the pair of guiding functions $\left(q^{(0)}(t), u^{(0)}(t)\right), t \geq t_{0}$, is replaced by a sequence of renewed pairs $\left(q^{(j)}(t), u^{(j)}(t)\right), t \geq t_{j}, j=1,2, \ldots$, of guiding functions determined by replanning, i.e. by repeated (OSTP) for the remaining time intervals $\left[t_{j}, t_{f}^{(j)}\right]$ and by using the new information given by $\mathcal{A}_{t_{j}}$. Since replanning at a later main correction time point $t_{j}, j \geq 1$, hence on-line, may be very time consuming, in order to maintain the real-time capability of the method, we may start the replanning procedure for an update of the guiding functions at time $t_{j}$ already at some earlier time $\tilde{t}_{j}$ with $t_{j-1}<\tilde{t}_{j}<t_{j}, j \geq 1$. Of course, in this case

$$
\mathcal{A}_{t_{j}}:=\mathcal{A}_{\tilde{t}_{j}}
$$

is defined to contain only the information about the control process up to time $\tilde{t}_{j}$ in which replanning for time $t_{j}$ starts.

Fig. 5.1 Start of replanning 
The resulting substitute problem at a stage $j \geq 1$ follows from the corresponding substitute problem for the previous stage $j-1$ just by updating $\zeta_{j-1} \rightarrow \zeta_{j}, \zeta_{f}^{(j-1)} \rightarrow \zeta_{f}^{(j)}$, the initial and terminal parameters, see (31a). The renewed

$$
\text { initial parameters } \zeta_{j}: t_{j}, s_{j}, \bar{q}_{j}, \overline{\dot{q}}_{j}, P_{p(\cdot)}^{(j)} \text { or } \nu_{j}
$$

for the $j$-th stage, $j \geq 1$, are determined recursively as follows:

$$
\begin{aligned}
s_{j}:= & s^{(j-1)}\left(t_{j}\right) \quad(1-1-\text { transformation } s=s(t)) \\
\bar{q}_{j}:= & \hat{q}\left(t_{j}\right), \bar{q}_{j}:=q^{(j-1)}\left(t_{j}\right) \text { or } \bar{q}_{j}:=E\left(q\left(t_{j}\right) \mid \mathcal{A}_{t_{j}}\right) \\
\bar{q}_{j}:= & \hat{\dot{q}}\left(t_{j}\right), \bar{q}_{j}:=\dot{q}^{(j-1)}\left(t_{j}\right) \text { or } \bar{q}_{j}:=E\left(\dot{q}\left(t_{j}\right) \mid \mathcal{A}_{t_{j}}\right) \\
& \left(\text { observation or estimate of } q\left(t_{j}\right), \dot{q}\left(t_{j}\right)\right) \\
P_{p(\cdot)}^{(j)}:= & P_{p(\cdot)}^{\left(t_{j}\right)}=P_{p(\cdot) \mid \mathcal{A}_{t_{j}}} \\
\nu_{j}:= & \nu^{\left(t_{j}\right)} .
\end{aligned}
$$

The renewed

$$
\text { terminal parameters } \zeta_{f}^{(j)}: t_{f}^{(j)}, s_{f}, \bar{q}_{f}^{(j)}, \beta_{f}
$$

for the $j$-th stage, $j \geq 1$, are defined by

$$
\begin{aligned}
& s_{f} \text { (given) } \\
& \bar{q}_{f}^{(j)}:=\hat{q}\left(t_{f}\right) \text { or } \bar{q}_{f}^{(j)}:=E\left(q_{f}(\omega) \mid \mathcal{A}_{t_{j}}\right) \\
& \beta_{f}=0 \\
& s^{(j)}\left(t_{f}^{(j)}\right)=s_{f},
\end{aligned}
$$

As already mentioned above, the (OSTP) for the $j$-th stage, $j \geq 1$, is obtained from the substitute problems (29a-f,f'), (32a-f), (33a-d), resp., formulated for the 0 -th stage, $j=0$, just by substituting

$$
\zeta_{0} \rightarrow \zeta_{j} \text { and } \zeta_{f} \rightarrow \zeta_{f}^{(j)}
$$

Let then denote

$$
\left(q_{e}^{(j)}, \beta^{(j)}\right)=\left(q_{e}^{(j)}(s), \beta^{(j)}(s)\right), s_{j} \leq s \leq s_{f}
$$

the corresponding pair of optimal solutions of the resulting substitute problem for the $j$-th stage, $j \geq 1$. 
The pair of guiding functions $\left(q^{(j)}(t), u^{(j)}(t)\right), t \geq t_{j}$, for the $j$-th stage, $j \geq 1$, is then defined as described in Section 4.2 for the 0-th stage. Hence, for the $j$-th stage, the reference trajectory in configuration space $q^{(j)}(t), t \geq t_{j}$, reads, cf. $(39 \mathrm{a})$

$$
q^{(j)}(t):=q_{e}^{(j)}\left(s^{(j)}(t)\right), t \geq t_{j},
$$

when the transformation $s^{(j)}:\left[t_{j}, t_{f}^{(j)}\right] \rightarrow\left[s_{j}, s_{f}\right]$ is defined by the initial value problem

$$
\dot{s}(t)=\sqrt{\beta^{(j)}(s)}, t \geq t_{j}, s^{(j)}\left(t_{j}\right)=s_{j} .
$$

The terminal time $t_{f}^{(j)}$ for the $j$-th stage is defined by the equation

$$
s^{(j)}\left(t_{f}^{(j)}\right)=s_{f}
$$

Moreover, again by the inverse dynamics approach, the feedforward control $u^{(j)}=u^{(j)}(t), t \geq t_{j}$, for the $j$-th stage is defined, see (40), (41), (42a,b), by

$$
u^{(j)}(t):=\Psi\left(v^{(j)}\left(t, p_{D}(\omega)\right) \mid \mathcal{A}_{t_{j}}\right),
$$

when

$$
v^{(j)}\left(t, p_{D}\right):=M\left(p_{D}, q^{(j)}(t)\right) \ddot{q}^{(j)}(t)+h\left(p_{D}, q^{(j)}(t), \dot{q}^{(j)}(t)\right), t \geq t_{j}
$$

Using the conditional expectation $\Psi\left(\cdot \mid \mathcal{A}_{t_{j}}\right):=E\left(\cdot \mid \mathcal{A}_{t_{j}}\right)$, we find the feedforward control

$$
u^{(j)}(t):=M\left(\bar{p}_{D}^{(j)}, q^{(j)}(t)\right) \ddot{q}^{(j)}(t)+h\left(\bar{p}_{D}^{(j)}, q^{(j)}, \dot{q}^{(j)}(t)\right), t \geq t_{j},
$$

where, cf. (31c),

$$
\bar{p}_{D}^{(j)}:=E\left(p_{D}(\omega) \mid \mathcal{A}_{t_{j}}\right)
$$

Corresponding to $(39 \mathrm{c}, \mathrm{d})$, the reference trajectory in work space $x^{(j)}=$ $x^{(j)}(t), t \geq t_{j}$, for the remaining time interval $t_{j} \leq t \leq t_{f}^{(j)}$, is defined by

$$
x^{(j)}(t):=E\left(T\left(p_{K}(\omega), q^{(j)}(t)\right) \mid \mathcal{A}_{t_{j}}\right)=T\left(\bar{p}_{K}^{(j)}, q^{(j)}(t)\right), t_{j} \leq t \leq t_{f}^{(j)},
$$

where

$$
\bar{p}_{K}^{(j)}:=E\left(p_{K}(\omega) \mid \mathcal{A}_{t_{j}}\right)
$$




\section{1. (OSTP)-transformation}

Since the (OSTP) on the differential stages $j=0,1,2 \ldots$ are determined uniquely by the set of initial and terminal parameters $\left(\zeta_{j}, \zeta_{f}^{(j)}\right)$, cf. (45a-f), (46a-e), these problems can be transformed to a reference problem depending on $\left(\zeta_{j}, \zeta_{f}^{(j)}\right)$ and a certain reference $s$-interval.

Theorem 5.1. Let $\left[\tilde{s}_{0}, \tilde{s}_{f}\right], \tilde{s}_{0}<\tilde{s}_{f}:=s_{f}$, be a given, fixed reference $s$-interval, and consider for a certain stage $j, j=0,1, \ldots$, the transformation

$$
\tilde{s}=\tilde{s}(s):=\tilde{s}_{0}+\frac{\tilde{s}_{f}-\tilde{s}_{0}}{s_{f}-s_{j}}\left(s-s_{j}\right), s_{j} \leq s \leq s_{f}
$$

from $\left[s_{j}, s_{f}\right]$ onto $\left[\tilde{s}_{0}, s_{f}\right]$ having the inverse

$$
s=s(\tilde{s})=s_{j}+\frac{s_{f}-s_{j}}{s_{f}-\tilde{s}_{0}}\left(\tilde{s}-\tilde{s}_{0}\right), \tilde{s}_{0} \leq \tilde{s} \leq \tilde{s}_{f} .
$$

Represent then the geometric path in work space $q_{e}=q_{e}(s)$ and the velocity profile $\beta=\beta(s), s_{j} \leq s \leq s_{f}$, for the $j$-th stage by

$$
\begin{aligned}
q_{e}(s) & :=\tilde{q}_{e}(\tilde{s}(s)), s_{j} \leq s \leq s_{f} \\
\beta(s) & :=\tilde{\beta}(\tilde{s}(s)), s_{j} \leq s \leq s_{f}
\end{aligned}
$$

where $\tilde{q}_{e}=\tilde{q}_{e}(\tilde{s}), \tilde{\beta}=\tilde{\beta}(\tilde{s}), \tilde{s}_{0} \leq \tilde{s} \leq \tilde{s}_{0} \leq \tilde{s} \leq \tilde{s}_{f}$, denote the corresponding functions on $\left[\tilde{s}_{0}, \tilde{s}_{f}\right]$. Then the (OSTP) for the $j$-th stage is transformed into a reference variational problem for $\left(\tilde{q}_{e}, \tilde{\beta}\right)$ depending on the parameters

$$
\left(\zeta, \zeta_{f}\right)=\left(\zeta_{j}, \zeta_{f}^{(j)}\right) \in Z \times Z_{f}
$$

and the reference $s$-interval $\left[\tilde{s}_{0}, \tilde{s}_{f}\right]$. Moreover, the optimal solution $\left(q_{e}^{(j)}, \beta^{(j)}\right)=$ $\left(q_{e}^{(j)}(s), \beta^{(j)}(s)\right), s_{j} \leq s \leq s_{f}$, may be represented by the optimal adaptive law

$$
\begin{aligned}
& q_{e}^{(j)}(s)=\tilde{q}_{e}^{*}\left(\tilde{s}(s) ; \zeta_{j}, \zeta_{f}^{(j)}\right), s_{j} \leq s \leq s_{f}, \\
& \beta^{(j)}(s)=\tilde{\beta}^{*}\left(\tilde{s}(s) ; \zeta_{j}, \zeta: f^{(j)}\right), s_{j} \leq s \leq s_{f},
\end{aligned}
$$

where

$$
\tilde{q}_{e}^{*}=\tilde{q}_{e}^{*}\left(\tilde{s} ; \zeta, \zeta_{f}\right), \tilde{\beta}^{*}=\tilde{\beta}^{*}\left(\tilde{s} ; \zeta, \zeta_{f}\right), \tilde{s}_{0} \leq \tilde{s} \leq \tilde{s}_{f},
$$

denotes the optimal solution of the above mentioned reference variational problem. 
Proof. According to $(53 \mathrm{a}, \mathrm{b})$ and $(52 \mathrm{a}, \mathrm{b})$, the derivatives of the functions $q_{e}(s), \beta(s), s_{j} \leq s \leq s_{f}$, are given by

$$
\begin{aligned}
& q_{e}^{\prime}(s)=\tilde{q}_{e}^{\prime}(\tilde{s}(s)) \frac{\tilde{s}_{f}-\tilde{s}_{0}}{s_{f}-s_{j}}, s_{j} \leq s \leq s_{f}, \\
& q_{e}^{\prime \prime}(s)=\tilde{q}_{e}^{\prime \prime}(\tilde{s}(s))\left(\frac{\tilde{s}_{f}-\tilde{s}_{0}}{s_{f}-s_{j}}\right)^{2}, s_{j} \leq s \leq s_{f}, \\
& \beta^{\prime}(s)=\tilde{\beta}^{\prime}(\tilde{s}(s)) \frac{\tilde{s}_{f}-\tilde{s}_{0}}{s_{f}-s_{j}}, s_{j} \leq s \leq s_{f} .
\end{aligned}
$$

Putting now the transformation $(52 \mathrm{a}, \mathrm{b})$ and the representation $(53 \mathrm{a}, \mathrm{b}),(56 \mathrm{a}-$ c) of $q_{e}(x), \beta(s), s_{j} \leq s \leq s_{f}$, and their derivatives into one of the substitute problems (29a-f,f'), (32a-f) or their time-variant versions, the chosen substitute problem is transformed into a corresponding reference variational problem with respect to the fixed reference interval $\left[\tilde{s}_{0}, \tilde{s}_{f}\right]$ and depending on the parameter vectors $\zeta_{j}, \zeta_{f}^{(j)}$. Moreover, according to $(53 \mathrm{a}, \mathrm{b})$, the optimal solution $\left(q_{e}^{(j)}, \beta^{(j)}\right)$ of the substitute problem for the $j$ - th stage may be represented then by (55a-c).

Remark 5.1. Based on the above theorem, the stage-independent functions $\tilde{q}_{e}^{*}, \tilde{\beta}^{*}$ can be determined now off-line by using an appropriate numerical procedure.

\subsection{The reference variational problem}

After the (OSTP)-transformation described in Section 5.1, in the time- invariant case for the problems of type (29a-f,f') we find

$$
\begin{array}{r}
\min \int_{\tilde{s}_{0}}^{\tilde{s}_{f}} E\left(L ^ { J } \left(p_{J}, \tilde{q}_{e}(\tilde{s},), \tilde{q}_{e}^{\prime}(\tilde{s}) \frac{\tilde{s}_{f}-\tilde{s}_{0}}{s_{f}-s_{j}}, \tilde{q}_{e}^{\prime \prime}(\tilde{s})\left(\frac{\tilde{s}_{f}-\tilde{s}_{0}}{s_{f}-s_{j}}\right)^{2}, \tilde{\beta}(\tilde{s}),\right.\right. \\
\left.\left.\tilde{\beta}^{\prime}(\tilde{s}) \frac{\tilde{s}_{f}-\tilde{s}_{0}}{s_{f}-s_{j}}\right) \mid \mathcal{A}_{t_{j}}\right) \frac{s_{f}-s_{j}}{\tilde{s}_{f}-\tilde{s}_{0}} d \tilde{s}+E\left(\phi^{J}\left(p_{J}, \tilde{q}_{e}\left(\tilde{s_{f}}\right) \mid \mathcal{A}_{t_{j}}\right)\right.
\end{array}
$$

s.t.

$$
\begin{aligned}
& E\left(f_{\gamma}\left(p, \tilde{q}_{e}(\tilde{s}), \tilde{q}_{e}^{\prime}(\tilde{s}) \frac{\tilde{s}_{f}-\tilde{s}_{0}}{s_{f}-s_{j}}, \tilde{q}_{e}^{\prime \prime}(\tilde{s})\left(\frac{\tilde{s}_{f}-\tilde{s}_{0}}{s_{f}-s_{j}}\right)^{2}, \tilde{\beta}(\tilde{s}), \tilde{\beta}^{\prime}(\tilde{s}) \frac{\tilde{s}_{f}-\tilde{s}_{0}}{s_{f}-s_{j}}\right) \mid \mathcal{A}_{t_{j}}\right) \leq \Gamma_{f}, \\
& \tilde{s}_{0} \leq \tilde{s} \leq \tilde{s}_{f} \\
& \tilde{\beta}(\tilde{s}) \geq 0, \tilde{s}_{0} \leq \tilde{s} \leq \tilde{s}_{f}
\end{aligned}
$$




$$
\begin{aligned}
& \tilde{q}_{e}\left(\tilde{s}_{0}\right)=\bar{q}_{j}, \tilde{q}_{e}^{\prime}\left(\tilde{s}_{0}\right) \frac{\tilde{s}_{f}-\tilde{s}_{0}}{s_{f}-s_{j}} \sqrt{\tilde{\beta}\left(\tilde{s}_{0}\right)}=\bar{q}_{j} \\
& \tilde{q}_{e}\left(\tilde{s}_{f}\right)=\bar{q}_{f}^{(j)}\left(\text { if } \phi^{J}=0\right), \tilde{\beta}\left(\tilde{s}_{f}\right)=0 \\
& \tilde{\beta}_{(}\left(\tilde{s}_{f}\right)=0, E\left(\psi\left(p, \tilde{q}_{e}\left(\tilde{s}_{f}\right)\right) \mid \mathcal{A}_{t_{j}}\right) \leq \Gamma_{\psi},
\end{aligned}
$$

where $f_{\gamma}, \Gamma_{f}$ are defined by

$$
f_{\gamma}:=\left(f_{\gamma}^{u}, g_{\gamma}^{u}, f_{\gamma}^{s}, g_{\gamma}^{s}\right)^{\prime}, \Gamma_{f}:=\left(\Gamma_{f}^{u}, \Gamma_{g}^{u}, \Gamma_{f}^{S}, \Gamma_{g}^{S}\right)^{\prime} .
$$

Moreover, for the problem type (32a-f) we get

$$
\begin{array}{r}
\min \int_{\tilde{s}_{0}}^{\tilde{s}_{f}} E\left(L _ { \gamma } ^ { J } \left(p, \tilde{q}_{e}(\tilde{s}), \tilde{q}_{e}^{\prime}(\tilde{s}) \frac{\tilde{s}_{f}-\tilde{s}_{0}}{s_{f}-s_{j}}, \tilde{q}_{e}^{\prime \prime}(\tilde{s})\left(\frac{\tilde{s}_{f}-\tilde{s}_{0}}{s_{f}-s_{j}}\right)^{2}, \tilde{\beta}(\tilde{s}),\right.\right. \\
\left.\left.\tilde{\beta}^{\prime}(\tilde{s}) \frac{\tilde{s}_{f}-\tilde{s}_{0}}{s_{f}-s_{j}}\right) \mid \mathcal{A}_{t_{j}}\right) \frac{s_{f}-s_{j}}{\tilde{s}_{f}-\tilde{s}_{0}} d \tilde{s}+E\left(\phi_{\gamma}^{J}\left(p, \tilde{q}_{e}\left(\tilde{s}_{f}\right)\right) \mid \mathcal{A}_{t_{j}}\right)
\end{array}
$$

s.t.

$$
\begin{aligned}
\tilde{\beta}(\tilde{s}) & \geq 0, \tilde{s}_{0} \leq \tilde{s} \leq \tilde{s}_{f} \\
\tilde{q}_{e}\left(\tilde{s}_{0}\right) & =\bar{q}_{j}, \tilde{q}_{e}^{\prime}\left(\tilde{s}_{0}\right) \frac{\tilde{s}_{f}-\tilde{s}_{0}}{s_{f}-s_{j}} \sqrt{\tilde{\beta}\left(\tilde{s}_{0}\right)}=\overline{\dot{q}}_{j} \\
\tilde{q}_{e}\left(\tilde{s}_{f}\right) & =\bar{q}_{f}^{(j)}\left(\text { if } \phi_{\gamma}^{J}=0\right), \tilde{\beta}\left(\tilde{s}_{f}\right)=0 .
\end{aligned}
$$

For the consideration of the time-variant case we note first that by using the transformation $(52 \mathrm{a}, \mathrm{b})$ and $(53 \mathrm{~b})$ the time $t \geq t_{j}$ can be represented, cf. (14a,b) and (15a), also by

$$
t=t\left(\tilde{s}, t_{j}, s_{j}, \tilde{\beta}(\cdot)\right):=t_{j}+\frac{s_{f}-s_{j}}{\tilde{s}_{f}-\tilde{s}_{0}} \int_{\tilde{s}_{0}}^{\tilde{s}} \frac{d \tilde{\sigma}}{\sqrt{\tilde{\beta}(\tilde{\sigma})}} .
$$

Hence, if the variational problems (57a-f) and (58a-d) for the $j$-th stage depend explicitly on time $t \geq t_{j}$, then, corresponding to (26a'-d'), for the constituting functions $L^{J}, \phi^{J}, L_{\gamma}^{J}, \phi_{\gamma}^{J}$ of the variational problems we have that

$$
\begin{aligned}
L^{J} & =L^{J}\left(\tilde{s}, t_{j}, s_{j}, \tilde{\beta}(\cdot), p_{J}, q_{e}, q_{e}^{\prime}, q_{e}^{\prime \prime}, \beta, \beta^{\prime}\right), \quad \tilde{s}_{0} \leq \tilde{s} \leq \tilde{s}_{f} \\
\phi^{J} & =\phi^{J}\left(\tilde{s}_{f}, t_{j}, s_{j}, \tilde{\beta}(\cdot), p_{J}, q_{e}\right) \\
f_{\gamma} & =f_{\gamma}\left(\tilde{s}, t_{j}, s_{j}, \tilde{\beta}(\cdot), p, q_{e}, q_{e}^{\prime}, q_{e}^{\prime \prime}, \beta, \beta^{\prime}\right), \quad \tilde{s}_{0} \leq \tilde{s} \leq \tilde{s}_{f} \\
L_{\gamma}^{J} & =L_{\gamma}^{J}\left(\tilde{s}, t_{j}, s_{j}, \tilde{\beta}(\cdot), p, q_{e}, q_{e}^{\prime \prime}, \beta, \beta^{\prime}\right), \quad \tilde{s}_{0} \leq \tilde{s} \leq \tilde{s}_{f} \\
\phi_{\gamma}^{J} & =\phi_{\gamma}^{J}\left(\tilde{s}_{f}, t_{j}, s_{j}, \tilde{\beta}(\cdot), p, q_{e}\right) .
\end{aligned}
$$




\subsubsection{Transformation of the initial state values}

Suppose here that $\phi^{J} \neq 0, \phi_{\gamma}^{J} \neq 0$, resp., and the terminal state condition (57e, e'), (58d), resp., is reduced to

$$
\tilde{\beta}\left(\tilde{s}_{f}\right)=0 .
$$

Representing then the unknown functions $\tilde{\beta}(\cdot), \tilde{q}_{e}(\cdot)$ on $\left[\tilde{s}_{0}, \tilde{s}_{f}\right]$ by

$$
\begin{gathered}
\tilde{\beta}(\tilde{s}):=\beta_{j} \tilde{\beta}_{a}(\tilde{s}), \quad \tilde{s}_{0} \leq \tilde{s} \leq \tilde{s}_{f} \\
\tilde{q}_{e}(\tilde{s}):=\bar{q}_{j d} \tilde{q}_{e a}(\tilde{s}), \quad \tilde{s}_{0} \leq \tilde{s} \leq \tilde{s}_{f},
\end{gathered}
$$

where $\bar{q}_{j d}$ denotes the diagonal matrix with the components of $\bar{q}_{j}$ on its main diagonal, then in terms of the new unknowns $\left(\tilde{\beta}_{a}(\cdot), \tilde{q}_{e a}(\cdot)\right)$ on $\left[\tilde{s}_{0}, \tilde{s}_{f}\right]$ we have the nonnegativity and fixed initial/terminal conditions

$$
\begin{aligned}
& \tilde{\beta}_{a}(\tilde{s}) \geq 0, \quad \tilde{s}_{0} \leq \tilde{s} \leq \tilde{s}_{f} \\
& \tilde{\beta}_{a}\left(\tilde{s}_{0}\right)=1, \quad \tilde{q}_{e a}\left(\tilde{s}_{0}\right)=\mathbf{1} \\
& \tilde{\beta}_{a}\left(\tilde{s}_{f}\right)=0,
\end{aligned}
$$

where $1:=(1, \ldots, 1)^{\prime}$.

\subsection{Numerical solutions of (OSTP) in real-time}

With the exception of field robots (e.g. Mars rover) and service robots [15], becoming increasingly important, the standard industrial robots move very fast. Hence, for industrial robots the optimal solution $\left(q_{e}^{(j)}(s), \beta^{(j)}(s)\right), \beta^{(j)}(s)$, resp., $s_{j} \leq s \leq s_{f}$, generating the renewed pair of guiding functions $\left(q^{(j)}(t), u^{(j)}(t)\right), t \geq t_{j}$, on each stage $j=1,2, \ldots$ should be provided in realtime. This means that the optimal solutions $\left(q_{e}^{(j)}, \beta^{(j)}\right), \beta^{(j)}$, resp., must be prepared off-line as far as possible such that on-line only relatively simple numerical operations are left.

Numerical methods capable to generate approximate optimal solutions in realtime are based mostly on discretization techniques, neural network (NN) approximation [3,25,26,33], linearization techniques (sensitivity analysis) [41]. 


\subsubsection{Discretization techniques}

Partitioning the space $Z \times Z_{f}$ of initial/terminal parameters $\left(\zeta, \zeta_{f}\right)$ into a certain (small) number $l_{0}$ of subdomains

$$
Z \times Z_{f}=\bigcup_{l=1}^{l_{0}} Z^{l} \times Z_{f}^{l}
$$

and selecting then a reference parameter vector

$$
\left(\zeta^{l}, \zeta_{f}^{l}\right) \in Z^{l} \times Z_{f}^{l}, l=1, \ldots, l_{0},
$$

in each subdomain $Z^{l} \times Z_{f}^{l}$, the optimal adaptive law (55c) can be approximated, cf. [40], by

$$
\left.\begin{array}{l}
\hat{\tilde{q}}_{e}^{*}\left(\tilde{s} ; \zeta, \zeta_{f}\right):=\tilde{q}^{*}\left(\tilde{s} ; \zeta^{l}, \zeta_{f}^{l}\right), \tilde{s}_{0} \leq \tilde{s} \leq \tilde{s}_{f} \\
\hat{\tilde{\beta}}^{*}\left(\tilde{s} ; \zeta, \zeta_{f}\right):=\tilde{\beta}^{*}\left(\tilde{s} ; \zeta^{l}, \zeta_{f}^{l}\right), \tilde{s}_{0} \leq \tilde{s} \leq \tilde{s}_{f}
\end{array}\right\} \text { for }\left(\zeta, \zeta_{f}\right) \in Z^{l} \times Z_{f}^{l}
$$

\subsubsection{NN-approximation}

For the determination of the optimal adaptive law (55a-c) in real-time, according to $(34 \mathrm{a}, \mathrm{b})$, the reference variational problem $(57 \mathrm{a}-\mathrm{f})$ or $(58 \mathrm{a}-\mathrm{d})$ is reduced first to a finite dimensional parameter optimization problem by

i) representing the unknown functions $\tilde{q}_{e}=\tilde{q}_{e}(\tilde{s}), \tilde{\beta}=\tilde{\beta}(\tilde{s}), \tilde{s}_{0} \leq \tilde{s} \leq \tilde{s}_{f}$, as linear combinations

$$
\begin{gathered}
\tilde{q}_{e}(\tilde{s}):=\sum_{l=1}^{l_{q}} \hat{q}_{e} B_{l}(\tilde{s}), \tilde{s}_{0} \leq \tilde{s} \leq \tilde{s}_{f}, \\
\tilde{\beta}\left(\tilde{s}:=\sum_{l=1}^{l_{\beta}} \hat{\beta}_{l} B_{l}^{\beta}(\tilde{s}), \tilde{s}_{0} \leq \tilde{s} \leq \tilde{s}_{f},\right.
\end{gathered}
$$

of certain basis functions, e.g. cubic B-splines, $B_{l}=B_{l}(\tilde{s}), B_{l}^{\beta}=B_{l}^{\beta}(\tilde{s}), \tilde{s}_{0} \leq$ $\tilde{s} \leq \tilde{s}_{f}, l=1, \ldots, l_{q}\left(l_{\beta}\right)$, with unknown vectorial (scalar) coefficients $\hat{q}_{l}, \hat{\beta}_{l}, l=$ $1, \ldots, l_{q}\left(l_{\beta}\right)$, and

ii) demanding the inequalities in $(57 \mathrm{~b}, \mathrm{c})$, (58b), resp., only for a finite set of $\tilde{s}$-parameter values $\tilde{s}_{0}<\tilde{s}_{1}<\ldots<\tilde{s}_{k}<\tilde{s}_{k+1}<\ldots<\tilde{s}_{\kappa}=\tilde{s}_{f}$.

By means of the above described procedure (i), (ii), the optimal coefficients

$$
\begin{aligned}
& \hat{q}_{l}^{*}=\hat{q}_{l}^{*}\left(\zeta, \zeta_{f}\right), l=1, \ldots, l_{q} \\
& \hat{\beta}_{l}^{*}=\hat{\beta}_{l}^{*}\left(\zeta, \zeta_{f}\right), l=1, \ldots, l_{\beta}
\end{aligned}
$$


become functions of the initial/terminal parameters $\zeta, \zeta_{f}, \mathrm{cf} . \quad(55 \mathrm{c}, \mathrm{d})$. Now, for the numerical realization of the optimal parameter functions $(63 \mathrm{c}, \mathrm{d})$, a Neural Network (NN) is emploid generating an approximative representation

$$
\begin{aligned}
& \hat{q}_{e}^{*}\left(\zeta, \zeta_{f}\right) \approx \hat{q}_{e}^{N N}\left(\zeta, \zeta_{f} ; w_{q}\right), l=1, \ldots, l_{q} \\
& \hat{\beta}_{l}^{*}\left(\zeta, \zeta_{f}\right) \approx \hat{\beta}_{l}^{N N}\left(\zeta, \zeta_{f} ; w_{\beta}\right), l=1, \ldots, l_{\beta},
\end{aligned}
$$

where the vectors $w_{q}, w_{\beta}$ of NN-weights are determined optimally

$$
w_{q}=w_{q}^{*}(\text { data }), w_{\beta}=w_{\beta}^{*}(\text { data })
$$

in an off-line training procedure $[3,25,33]$. Here, the model $(64 a, b)$ is fitted in the LSQ-sense to given data

$$
\left(\begin{array}{c}
\zeta^{\tau}, \zeta_{f}^{\tau} \\
\hat{q}_{l}^{* \tau}, l=1, \ldots, l_{q}
\end{array}\right),\left(\begin{array}{c}
\zeta^{\tau}, \zeta_{f}^{\tau} \\
\hat{\beta}_{l}^{* \tau}, l=1, \ldots, l_{\beta}
\end{array}\right), \tau=1, \ldots, \tau_{0},
$$

where

$$
\left(\zeta^{\tau}, \zeta_{f}^{\tau}\right), \tau=1, \ldots, \tau_{0},
$$

is a certain collection of initial/terminal parameter vectors, and

$$
\begin{aligned}
& \hat{q}_{l}^{* \tau}:=\hat{q}_{l}^{* \tau}\left(\zeta^{\tau}, \zeta_{f}^{\tau}\right), l=1, \ldots, l_{q}, \tau=1, \ldots, \tau_{0} \\
& \hat{\beta}_{l}^{* \tau}:=\hat{\beta}_{l}^{* \tau}\left(\zeta^{\tau}, \zeta_{f}^{\tau}\right), l=1, \ldots, l_{\beta}, \tau=1, \ldots, \tau_{0}
\end{aligned}
$$

are the related optimal coefficients in $(63 \mathrm{a}, \mathrm{b})$ which are determined off-line by an appropriate parameter optimization procedure.

Having the vectors $w_{q}^{*}, w_{\beta}^{*}$ of optimal NN-weights, by means of $(65 \mathrm{a}-\mathrm{c})$, for given actual initial/terminal parameters $\left(\zeta, \zeta_{f}\right)=\left(\zeta_{j}, \zeta_{f}^{(j)}\right)$ at stage $j \geq 0$, the NN yields then the optimal parameters

$$
\hat{q}_{l}^{*}\left(\zeta_{j}, \zeta_{f}^{(j)}\right), \hat{\beta}_{l}^{*}\left(\zeta_{j}, \zeta_{f}^{(j)}\right), l=1, \ldots, l_{q}\left(l_{\beta}\right)
$$

in real-time; consequently, by means of $(63 \mathrm{c}, \mathrm{d})$, also the optimal functions $\tilde{q}_{e}^{*}(\tilde{s}), \tilde{\beta}^{*}(\tilde{s}), \tilde{s}_{0} \leq \tilde{s} \leq \tilde{s}_{f}$, are then available very fast. For more details, see $[3,25]$.

\subsubsection{Linearization methods}

I) Linearization of the optimal feedforward control law 
Expanding the optimal control laws (55c) with respect to the initial/terminal parameter vector $\hat{\zeta}=\left(\zeta, \zeta_{f}\right)$ at its value $\hat{\zeta}_{0}=\left(\zeta_{0}, \zeta_{f}^{(0)}\right)$ for stage $j=0$, approximatively we have that

$$
\begin{aligned}
& \tilde{q}_{e}^{*}\left(\tilde{s}, \zeta, \zeta_{f}\right)=\tilde{q}_{e}^{*}\left(\tilde{s} ; \zeta_{0}, \zeta_{f}^{(0)}\right)+\frac{\partial \tilde{q}_{e}^{*}}{\partial \hat{\zeta}}\left(\tilde{s} ; \zeta_{0}, \zeta_{f}^{(0)}\right)\left(\zeta-\zeta_{0}, \zeta_{f}-\zeta_{f}^{(0)}\right)+\ldots(65 \mathrm{a}) \\
& \tilde{\beta}^{*}\left(\tilde{s} ; \zeta, \zeta_{f}\right)=\tilde{\beta}^{*}\left(\tilde{s} ; \zeta_{0}, \zeta_{f}^{(0)}\right)+\frac{\partial \tilde{\beta}^{*}}{\partial \hat{\zeta}}\left(\tilde{s} ; \zeta_{0}, \zeta_{f}^{(0)}\right)\left(\zeta-\zeta_{0}, \zeta_{f}-\zeta_{f}^{(0)}\right)+\ldots(65 \mathrm{~b})
\end{aligned}
$$

where the optimal starting functions $\tilde{q}_{e}^{*}\left(\tilde{s} ; \zeta_{0}, \zeta_{f}^{(0)}\right), \tilde{\beta^{*}}\left(\tilde{s} ; \zeta_{0}, \zeta_{f}^{(0)}\right)$ and the derivatives $\frac{\partial \tilde{\beta}^{*}}{\partial \hat{\zeta}}\left(\tilde{s} ; \zeta_{0}, \zeta_{f}^{(0)}\right), \frac{\partial \tilde{\beta}^{*}}{\partial \hat{\zeta}}\left(\tilde{s} ; \zeta_{0}, \zeta_{f}^{(0)}\right), \ldots$ can be determined - on a certain grid of $\left[\tilde{s}_{0}, \tilde{s}_{f}\right]$ - off-line by using sensitivity analysis [41]. The actual values of $\tilde{q}_{e}^{*}, \tilde{\beta}^{*}$ at later stages can be obtained then very rapidly by means of simple matrix ope- rations. If necessary, the derivatives can be updated later on by a numerical procedure running in parallel to the control process.

II) Sequential linearization of the (AOSTP) process

Given the optimal guiding functions $q_{e}^{(j)}=q_{e}^{(j)}(s), \beta^{(j)}=\beta^{(j)}(s), s_{j} \leq s \leq s_{f}$ for the $j$-th stage, corresponding to the representation $(35 \mathrm{a}, \mathrm{b})$, the optimal guiding functions $q_{e}^{(j+1)}(s), \beta^{(j+1)}(s), s_{j+1} \leq s \leq s_{f}$, are represented by

$$
\begin{array}{ll}
q_{e}^{(j+1)}(s):=q_{e}^{(j)}(s)+\Delta q_{e}(s), & s_{j+1} \leq s \leq s_{f}, \\
\beta^{(j+1)}(s):=\beta^{(j)}(s)+\Delta \beta(s), & s_{j+1} \leq s \leq s_{f}
\end{array}
$$

where $s_{j}<s_{j+1}<s_{f}$, and $\left(\Delta q_{e}(s), \Delta \beta(s)\right), s_{j+1} \leq s \leq s_{f}$, are certain (small) changes of the $j$-th stage optimal guiding functions $\left(q_{e}^{(j)}(\cdot), \beta^{(j)}(\cdot)\right)$. 
Fig. 5.2 Sequential linearization of (AOSTP)

Obviously, the linearization technique described in Section 4.1c can be applied now also to the approximate computation of the optimal changes $\Delta q_{e}(s), \Delta \beta(s), s_{j+1} \leq s \leq s_{f}$, if the following replacements are made in the formulas $(35 \mathrm{a}, \mathrm{b}),(36 \mathrm{a}-\mathrm{c}),(37 \mathrm{a}-\mathrm{h})$ and $(38 \mathrm{a}-\mathrm{e})$ :

$$
\left.\begin{array}{ll}
s_{0} \leq s \leq s_{f} & \rightarrow s_{j+1} \leq s \leq s_{f} \\
\bar{q}_{e}, \bar{\beta} & \rightarrow q_{e}^{(j)}, \beta^{(j)} \\
\mathcal{A}_{t_{0}} & \rightarrow \mathcal{A}_{t_{j+1}} \\
\bar{g}^{(0)}, \bar{\phi}^{(0)}, \bar{F}^{(0)} & \rightarrow \bar{g}^{(j+1)}, \bar{\phi}^{(j+1)}, \bar{F}^{(j+1)} \\
\bar{A}_{g, \bar{q}_{e}, \bar{\beta}}^{(0)}, \ldots, \bar{H}_{g, \bar{q}_{e}, \bar{\beta}}^{(0)} & \rightarrow \bar{A}_{g, q_{e}^{(j)}, \beta(j)}^{(j+1)}, \bar{H}_{g, q_{e}^{(j)}, \beta(j)}^{(j+1)} \\
\Delta \beta\left(s_{0}\right)=0 & \rightarrow \Delta \beta\left(s_{j+1}\right)=\beta_{j+1}-\beta^{(j)}\left(s_{j+1}\right) \\
\Delta q_{e}\left(s_{0}\right)=q_{0}-\bar{q}_{e}\left(s_{0}\right) & \rightarrow \Delta q_{e}\left(s_{j+1}\right)=\bar{q}_{j+1}-q_{e}^{(j)}\left(s_{j+1}\right) \\
\Delta \beta\left(s_{f}\right)=0 & \rightarrow \Delta \beta\left(s_{f}\right)=0 \\
\Delta q_{e}\left(s_{f}\right)=q_{f}^{(0)}-\bar{q}_{e}\left(s_{f}\right) & \rightarrow \Delta q_{e}\left(s_{f}\right)=\bar{q}_{f}^{(j+1)}-\bar{q}_{f}^{(j)}, \text { if } \phi^{J}=0,
\end{array}\right\}
$$

where $\bar{X}^{(j+1)}$ denotes the conditional expectation with respect to $\mathcal{A}_{t_{j+1}}$, cf. (50d), (51b). Furthermore, (38f) yields

$$
\left(q_{e}^{(j)^{\prime}}\left(s_{j+1}\right)+\Delta q_{e}^{\prime}\left(s_{j+1}\right)\right) \cdot \sqrt{\beta_{j+1}}=\bar{q}_{j+1}
$$


which can be approximated, cf. (38f'), by

$$
\sqrt{\beta^{(j)}\left(s_{j+1}\right)} \Delta q_{e}^{\prime}\left(s_{j+1}\right)+\frac{1}{2} \frac{\Delta \beta\left(s_{j+1}\right)}{\sqrt{\beta^{(j)}\left(s_{j+1}\right)}} q_{e}^{(j)^{\prime}}\left(s_{j+1}\right) \approx \overline{\dot{q}}_{j+1}-\sqrt{\beta^{(j)}\left(s_{j+1}\right)} q_{e}^{(j)^{\prime}}\left(s_{j+1}\right) .
$$

Depending on the chosen substitute problem, by this linearization method we obtain then a variational problem, an optimization problem, resp., for the changes $\left(\Delta q_{e}(s), \Delta \beta(s)\right), s_{j+1} \leq s \leq s_{f}$, having a linear objective function and/or linear constraints.

To give a typical example, we consider now (AOSTP) on the $(j+1)$ th stage with substitute problem (32a-f). Hence, the functions $g, \phi$ in (36a-c), (37a-h), and (38 a-e) are given by

$$
g:=L_{\gamma}^{J}, \phi:=\phi_{\gamma}^{J}
$$

Applying the linearization techniques developped in Section 4.1c now to (32a-f), according to (38a-c), (37b) and (66d'), for the correction terms $\Delta q_{e}(s), \Delta \beta(s), s_{j+1} \leq s \leq s_{f}$, we find the following linear optimization problem:

$$
\begin{array}{r}
\min \int_{s_{j+1}}^{s_{f}} \bar{G}_{g, q_{e}^{(j)}, \beta(j)}^{(j+1)}(s)^{T} \Delta q_{e}(s) d s+\int_{s_{j+1}}^{s_{f}} \bar{H}_{g, q_{e}, \beta(j)}^{(j+1)}(s) \Delta(s) d s \\
+R_{j}^{T} \Delta q_{e}\left(s_{f}\right)+S_{j}^{T} \Delta q_{e}^{\prime}\left(s_{f}\right)+T_{j} \Delta \beta\left(s_{j+1}\right)
\end{array}
$$

s.t.

$$
\begin{aligned}
\Delta q_{e}\left(s_{j+1}\right) & =\bar{q}_{j+1}-q_{e}^{(j)}\left(s_{j+1}\right) \\
\Delta q_{e}\left(s_{f}\right) & =\bar{q}_{f}^{(j+1)}-\bar{q}_{f}^{(j)}, \text { if } \phi^{J}=0 \\
\Delta \beta\left(s_{f}\right) & =0 \\
\Delta \beta(s) & \geq-\beta^{(j)}(s), s_{j+1} \leq s \leq s_{f},
\end{aligned}
$$

where

$$
\begin{aligned}
R_{j} & :=\bar{B}_{g, q_{e}^{(j)}, \beta(j)}^{(j+1)}\left(s_{f}\right)-\bar{C}_{g, q_{e}^{(j)}, \beta(j)}^{(j+1)}\left(s_{f}\right)+\bar{a}_{\phi, q_{e}^{(j)}}^{(j+1)}\left(s_{f}\right) \\
S_{j} & :=\bar{C}_{g, q_{e}^{(j)}, \beta^{(j)}}^{(j+1)}\left(s_{f}\right) \\
T_{j} & :=\frac{1}{2} \bar{C}_{g, q_{e}^{(j)}, \beta^{(j)}}^{(j+1)}\left(s_{j+1}\right)^{T} \frac{q_{e}^{(j)^{\prime}}\left(s_{j+1}\right)}{\beta^{(j)}\left(s_{j+1}\right)}-\bar{E}_{g, q_{e}^{(j)}, \beta^{(j)}}^{(j+1)}\left(s_{j+1}\right) .
\end{aligned}
$$


The linear optimization problem $(67 \mathrm{a}-\mathrm{g})$ can be solved now by the methods developed e.g. in [12], where for the correction terms $\Delta q_{e}(s), \Delta \beta(s), s_{j+1} \leq s \leq$ $s_{f}$, some box constraints or norm bounds have to be added to (67a-g). In case of $\Delta \beta(\cdot)$ we may replace, e.g., (67d) by the condition

$$
-\beta^{(j)}(s) \leq \Delta \beta(s) \leq \Delta \beta^{\max }, s_{j+1} \leq s \leq s_{f},
$$

with some upper bound $\Delta \beta^{\max }$.

It is easy to see that $(67 \mathrm{a}-\mathrm{g})$ can be split up into two separated linear optimization problems for $\Delta q_{e}(\cdot), \Delta \beta(\cdot)$, respectively. Hence, according to the simple structure of the objective function (67a), we observe that

$$
\operatorname{sign}\left(\bar{H}_{g, q_{e}^{(j)}, \beta(j)}^{(j+1)}(s)\right), s_{j+1} \leq s \leq s_{f}, \text { and } \operatorname{sign}\left(T_{j}\right)
$$

indicates the points $s$ in the interval $\left[s_{j+1}, s_{f}\right]$ with $\Delta \beta(s)<0$ or $\Delta \beta(s)>0$, hence, the points $s, s_{j+1} \leq s \leq s_{f}$, where the velocity profile should be decreased/increased. Moreover, using (67d'), the optimal correction $\Delta \beta(s)$ is equal to the lower/upper bound in (67d') depending on the above mentioned signs.

Obviously, the correction vectors $\Delta q_{e}(s), s_{j+1} \leq s \leq s_{f}$, for the geometric path in configuration space can be determined in the same way. Similar results are obtained also if we use $L_{2}$-norm bounds for the correction terms.

If the pointwise constraints $(29 \mathrm{~b}, \mathrm{c})$ in (49a-f, f') are averaged with respect to $s, s_{j+1} \leq s \leq s_{f}$, then functions of the type (36c) arise, cf. (29b', $\left.\mathrm{c}^{\prime}\right)$, which can be linearized again by the same techniques as discussed above. In this case, linear constraints are obtained for $\Delta \beta(s), \Delta q_{e}(s), s_{j+1} \leq s \leq s_{f}$, with constrant functions of the type (38a-c), cf. also (67a).

\subsubsection{Combination of discretization and linearization}

Obviously, the methods described briefly in Sections 5.3.1 and 5.3.2 can be combined in the following way:

First, by means of discretization (Finite Element Methods), an approximate optimal control law $\left(\tilde{q}_{e}^{*}, \beta^{*}\right)$ is searched in a class of finitely generated functions of the type $(63 \mathrm{a}, \mathrm{b})$. Corresponding to $(65 \mathrm{a}, \mathrm{b})$, by means of Taylor expansion 
here the optimal coefficients $\hat{q}_{l}^{*}, \hat{\beta}_{l}^{*}, l=1, \ldots, l_{q}\left(l_{\beta}\right)$, in the corresponding linear combination of type $(63 \mathrm{a}, \mathrm{b})$ are represented, $\mathrm{cf}$. $(63 \mathrm{c}, \mathrm{d})$, by

$$
\begin{aligned}
& \hat{q}_{l}^{*}\left(\zeta, \zeta_{f}\right)=\hat{q}_{l}^{*}\left(\zeta_{0}, \zeta_{f}^{(0)}\right)+\frac{\partial \hat{q}_{l}^{*}}{\partial \hat{\zeta}}\left(\zeta_{0}, \zeta_{f}^{(0)}\right)\left(\zeta-\zeta_{0}, \zeta_{f}-\zeta_{f}^{(0)}\right)+\ldots, \\
& \hat{\beta}_{l}^{*}\left(\zeta, \zeta_{f}\right)=\hat{\beta}_{l}^{*}\left(\zeta_{0}, \zeta_{f}^{(0)}\right)+\frac{\partial \hat{\beta}_{l}^{*}}{\partial \hat{\zeta}}\left(\zeta_{0}, \zeta_{f}^{(0)}\right)\left(\zeta-\zeta_{0}, \zeta_{f}-\zeta_{f}^{(0)}\right)+\ldots
\end{aligned}
$$

$l=1, \ldots, l_{q}\left(l_{\beta}\right)$. Here, the derivatives

$$
\frac{\partial \hat{q}_{l}^{*}}{\partial \hat{\zeta}}\left(\zeta_{0}, \zeta_{f}^{(0)}\right), \frac{\partial \hat{\beta}_{l}^{*}}{\partial \hat{\zeta}}\left(\zeta_{0}, \zeta_{f}^{(0)}\right), \ldots, l=1, \ldots, l_{q}\left(l_{\beta}\right),
$$

can be determined again by sensitivity analysis [41] of a finite dimensional parameter-dependent optimization problem which may be much simpler than the sensitivity analysis of the parameter-dependent variational problem (57a-f) or $(58 \mathrm{a}-\mathrm{d})$.

Stating the necessary (and under additional conditions also sufficient) KuhnTucker conditions for the optimal coefficients $\hat{q}_{l}^{*}, \hat{\beta}_{l}^{*}, l=1, \ldots, l_{q}\left(l_{\beta}\right)$, formulas for the derivatives $(68 \mathrm{c})$ may be obtained by partial differentiation with respect to the complete vector $z=\left(\zeta, \zeta_{f}\right)$ of initial/terminal parameters.

\section{On-line control corrections}

We consider now the control of the robot at the $j$-th stage, i.e. for time $t \geq t_{j}$. Due to random variations of the vector $p$ of the model parameters of the robot and its environment, and due to possible deviations of the true initial state $\left(q_{j}, \dot{q}_{j}\right):=\left(q\left(t_{j}\right), \dot{q}\left(t_{j}\right)\right)$ in configuration space from the corresponding initial values $\left(\bar{q}_{j}, \bar{q}_{j}\right)=\left(\bar{q}_{j}, q_{e}^{(j)}\left(s_{j}\right) \sqrt{\beta_{j}}\right)$ of the (OSTP) at stage $j$, there is a remaining deviation of the actual trajectory

$$
q(t)=q\left(t, p_{D}, q_{j}, \dot{q}_{j}, u(\cdot)\right), t \geq t_{j}
$$

in configuration space of the robot from the optimal reference trajectory

$$
q^{(j)}(t)=q_{e}^{(j)}\left(s^{(j)}(t)\right)=q\left(t, \bar{p}_{D}^{(j)}, \bar{q}_{j}, \bar{q}_{j} \cdot u^{(j)}(\cdot)\right),
$$

see $(44 a, b),(45 a-f),(49 a, b)$ and (50c). In the following we assume that the state $(q(t), \dot{q}(t))$ in configuration space may be observed for $t>t_{j}$. In oder to define 
now an appropriate control correction (feedback control law), see (2) and Fig. 6.1 ,

$$
\Delta u^{(j)}(t)=u(t)-u^{(j)}(t):=\varphi^{(j)}\left(t, \Delta z^{(j)}(t)\right), t \geq t_{j},
$$

for the compensation of the tracking error

$$
\Delta z^{(j)}(t):=z(t)-z^{(j)}(t), z(t):=\left(\begin{array}{c}
q(t) \\
\dot{q}(t)
\end{array}\right), z^{(j)}(t):=\left(\begin{array}{c}
q^{(j)}(t) \\
\dot{q}^{(j)}(t)
\end{array}\right),
$$

where $\varphi^{(j)}=\varphi^{(j)}(t, \Delta q, \dot{\Delta} q)$ is a function such that

$$
\varphi^{(j)}(t, 0,0)=0 \text { for all } t \geq t_{j},
$$

the trajectories $q(t)$ and $q^{(j)}(t), t \geq t_{j}$, are embedded into a one-parameter family of trajectories $q=q(t, \epsilon), t \geq t_{j}, 0 \leq \epsilon \leq 1$, in configuration space which are defined as follows:

Consider first the following initial data for stage $j$ :

$$
\begin{aligned}
q_{j}(\epsilon) & :=\bar{q}_{j}+\epsilon \Delta q_{j}, \Delta q_{j}:=q_{j}-\bar{q}_{j} \\
\dot{q}_{j}(\epsilon) & :=\overline{\dot{q}}_{j}+\epsilon \dot{\Delta} q_{j}, \dot{\Delta} q_{j}:=\dot{q}_{j}-\overline{\dot{q}}_{j} \\
p_{D}(\epsilon) & :=\bar{p}_{D}^{(j)}+\epsilon \Delta p_{D}, \Delta p_{D}:=p_{D}-\bar{p}_{D}^{(j)}, 0 \leq \epsilon \leq 1 .
\end{aligned}
$$

Moreover, define the control input $u(t), t \geq t_{j}$, by (70a), hence,

$$
\begin{aligned}
u(t) & =u^{(j)}(t)+\Delta u^{(j)}(t) \\
& =u^{(j)}(t)+\varphi^{(j)}\left(t, q(t)-q^{(j)}(t), \dot{q}(t)-\dot{q}^{(j)}(t)\right), t \geq t_{j} .
\end{aligned}
$$

Let then denote

$$
q(t, \epsilon)=q\left(t, p_{D}(\epsilon), q_{j}(\epsilon), \dot{q}_{j}(\epsilon), u(\cdot)\right), 0 \leq \epsilon \leq 1, t \geq t_{j},
$$

the solution of the following initial value problem consisting of the dynamic equation (4a) with the initial values, the vector of dynamic parameters and the total control input $u(t)$ given by (71a-d):

$$
F\left(p_{D}(\epsilon), q(t, \epsilon), \dot{q}(t, \epsilon), \ddot{q}(t, \epsilon)\right)=u(t, \epsilon), 0 \leq \epsilon \leq 1, t \geq t_{j}
$$

where

$$
\begin{aligned}
q\left(t_{j}, \epsilon\right) & =q_{j}(\epsilon), \dot{q}\left(t_{j}, \epsilon\right)=\dot{q}_{j}(\epsilon), \\
u(t, \epsilon) & :=u^{(j)}(t)+\varphi^{(j)}\left(t, q(t, \epsilon)-q^{(j)}(t), \dot{q}(t, \epsilon)-\dot{q}^{(j)}(t)\right),
\end{aligned}
$$


and $F=F\left(p_{D}, q, \dot{q}, \ddot{q}\right)$ is defined, cf. (4a), by

$$
F\left(p_{D}, q, \dot{q}, \ddot{q}\right):=M\left(p_{D}, q\right) \ddot{q}+h\left(p_{D}, q, \dot{q}\right) .
$$

In the following we suppose [19] that the initial value problem (73a-d) has a unique solution $q=q(t, \epsilon), t \geq t_{j}$, for each parameter value $\epsilon, 0 \leq \epsilon \leq 1$. 
6.1. Basic properties of the embedding $q(t, \epsilon)$

6.1.1. $\epsilon=\epsilon_{0}:=0$

Because of condition (70c) of the feedback control law $\varphi^{(j)}$ to be determined, and due to the unique solvability assumption of the initial value problem (73a-d) at the $j$-th stage, for $\epsilon=0$ we have that

$$
q(t, 0)=q^{(j)}(t), t \geq t_{j} .
$$

6.1.2. $\epsilon=\epsilon_{1}:=1$

According to (69a), (70a-c) and (71a-d),

$$
q(t, 1)=q(t)=q\left(t, p_{D}, q_{j}, \dot{q}_{j}, u(\cdot)\right), t \geq t_{j},
$$

denotes the actual trajectory in configuration space under the total control input $u(t)=u^{(j)}(t)+\Delta u^{(j)}(t), t \geq t_{j}$, given by $(71 \mathrm{~d})$.

\subsubsection{Taylor-expansion with respect to $\epsilon$}

Let $\Delta \epsilon=\epsilon_{1}-\epsilon_{0}=1$, and suppose that the following property known from parameter-dependent differential equations, cf. [19], holds:

Assumption 6.1. The solution $q=q(t, \epsilon), t \geq t_{j}, 0 \leq \epsilon \leq 1$, of the initial value problem (71a-d) has continuous derivatives with respect to $\epsilon$ up to order $\nu>1$ for all $t_{j} \leq t \leq t_{j}+\Delta t_{j}, 0 \leq \epsilon \leq 1$, with a certain $\Delta t_{j}>0$.

Note that $(t, \epsilon) \rightarrow q(, \epsilon), t \geq t_{j}, 0 \leq \epsilon \leq 1$, can be interpreted as a homotopy from the reference trajectory $q^{(j)}(t)$ to the actual trajectory $q(t), t \geq t_{j}$, cf. [34].

Based on the above assumption and (74a,b), by Taylor expansion with respect to $\epsilon$ at $\epsilon=\epsilon_{0}=0$, the actual trajectory of the robot can be represented by

$$
\begin{aligned}
q(t) & =q\left(t, p_{D}, q_{j}, \dot{q}_{j}, u(\cdot)\right)=q(t, 1)=q\left(t, \epsilon_{0}+\Delta \epsilon\right) \\
& =q(t, 0)+\Delta q(t)=q^{(j)}(t)+\Delta q(t),
\end{aligned}
$$

where the expansion of the tracking error $\Delta q(t), t \geq t_{j}$, is given by

$$
\Delta q(t)=\sum_{l=1}^{\nu-1} \frac{1}{l !} d^{l} q(t)(\Delta \epsilon)^{l}+\frac{1}{\nu !} \frac{\partial^{\nu} q}{\partial \epsilon^{\nu}}(t, \vartheta)(\Delta \epsilon)^{\nu}
$$




$$
=\sum_{l=1}^{\nu-1} \frac{1}{l !} d^{l} q(t)+\frac{1}{\nu !} \frac{\partial^{\nu} q}{\partial \epsilon^{\nu}}(t, \vartheta), t \geq t_{j} .
$$

Here, $\vartheta=\vartheta(t, \nu), 0<\vartheta<1$, and

$$
d^{l} q(t):=\frac{\partial^{l} q}{\partial \epsilon^{l}}(t, 0), t \geq t_{j}, l=1,2, \ldots,
$$

denote the $l$-th order differentials of $q=q(t, \epsilon)$ with respect to $\epsilon$ at $\epsilon=\epsilon_{0}=0$. Obviously, differential equations for the differentials $d^{l} q(t), l=1,2, \ldots$, may be obtained, cf. [19], by successive differentiation of the initial value problem (73a-d) with respect to $\epsilon$ at $\epsilon_{0}=0$.

Furthermore, based on the Taylor expansion of the tracking error $\Delta q(t), t \geq$ $t_{j}$, using some stability requirements, the tensorial coefficients $D_{z}^{l} \varphi^{(j)}(t, 0), l=$ $1,2, \ldots$, of the Taylor expansion

$$
\varphi^{(j)}(t, \Delta z)=\sum_{l=1}^{\infty} \mathbf{D}_{z}^{l} \varphi^{(j)}(t, 0) \cdot(\Delta z)^{l}
$$

of the feedback control law $\varphi^{(j)}=\varphi^{(j)}(t, \Delta z)$ can be determined at the same time.

\subsection{The 1st order differential $d q$}

Next to we have to introduce some definitions. Corresponding to (70b) and (72) we put

$$
z(t, \epsilon):=\left(\begin{array}{c}
q(t, \epsilon) \\
\dot{q}(t, \epsilon)
\end{array}\right), t \geq t_{j}, 0 \leq \epsilon \leq 1 ;
$$

then, we define the following Jacobians of the function $F$ given by (73d):

$$
\begin{aligned}
K\left(p_{D}, q, \dot{q}, \ddot{q}\right) & :=F_{q}\left(p_{D}, q, \dot{q}, \ddot{q}\right)=\mathbf{D}_{q} F\left(p_{D}, q, \dot{q}, \ddot{q}\right) \\
D\left(p_{D}, q, \dot{q}\right) & :=F_{\dot{q}}\left(p_{D}, q, \dot{q}, \ddot{q}\right)=h_{\dot{q}}\left(p_{D}, q, \dot{q}\right) .
\end{aligned}
$$

Moreover, it is

$$
M\left(p_{D}, q\right)=F_{\ddot{q}}\left(p_{D}, q, \dot{q}, \ddot{q}\right),
$$

and due to the linear parametrization property of robots, see Remark $2.2, F$ may be represented by

$$
F\left(p_{D}, q, \dot{q}, \ddot{q}\right)=Y(q, \dot{q}, \ddot{q}) p_{D}
$$


with a certain matrix function $Y=Y(q, \dot{q}, \ddot{q})$.

By differentiation of (73a-d) with respect to $\epsilon$, for the partial derivative $\frac{\partial q}{\partial \epsilon}(t, \epsilon)$ of $q=q(t, \epsilon)$ with respect to $\epsilon$ we find, cf. (70b), the following linear initial value problem (error differential equation)

$$
\begin{aligned}
& Y(q(t, \epsilon), \dot{q}(t, \epsilon), \ddot{q}(t, \epsilon)) \Delta p_{D}+K\left(p_{D}(\epsilon), q(t, \epsilon), \dot{q}(t, \epsilon), \ddot{q}(t, \epsilon)\right) \frac{\partial q}{\partial \epsilon}(t, \epsilon) \\
+ & D\left(p_{D}(\epsilon), q(t, \epsilon), \dot{q}(t, \epsilon)\right) \frac{d}{d t} \frac{\partial q}{\partial \epsilon}(t, \epsilon)+M\left(p_{D}(\epsilon), q(t, \epsilon)\right) \frac{d^{2}}{d t^{2}} \frac{\partial q}{\partial \epsilon}(t, \epsilon) \\
= & \frac{\partial u}{\partial \epsilon}(t, \epsilon)=\frac{\partial \varphi^{(j)}}{\partial z}\left(t, \Delta z^{(j)}(t)\right) \frac{\partial z}{\partial \epsilon}(t, \epsilon)
\end{aligned}
$$

with the initial values, see (71a,b),

$$
\frac{\partial q}{\partial \epsilon}\left(t_{j}, \epsilon\right)=\Delta q_{j}, \frac{d}{d t} \frac{\partial q}{\partial \epsilon}\left(t_{j}, \epsilon\right)=\dot{\Delta} q_{j}
$$

Putting now $\epsilon=\epsilon_{0}=0$, because of $(70 \mathrm{a}, \mathrm{b})$ and $(74 \mathrm{a})$, system $(77 \mathrm{a}, \mathrm{b})$ yields then this system of 2 nd order differential equations for the 1 st order differential $d q(t)=\frac{\partial q}{\partial \epsilon}(t, 0):$

$$
\begin{aligned}
& Y^{(j)}(t) \Delta p_{D}+K^{(j)}(t) d q(t)+D^{(j)}(t) \dot{d} q(t)+M^{(j)}(t) \ddot{d} q(t) \\
& \quad=d u(t)=\varphi_{z}^{(j)}(t, 0) d z(t)=\varphi_{q}^{(j)}(t, 0) d q(t)+\varphi_{\dot{q}}^{(j)}(t, 0) \dot{d} q(t), t \geq t_{j},
\end{aligned}
$$

with the initial values

$$
d q\left(t_{j}\right)=\Delta q_{j}, \dot{d} q\left(t_{j}\right)=\dot{\Delta} q_{j}
$$

Here,

$$
\begin{aligned}
& d u(t):=\frac{\partial u}{\partial \epsilon}(t, 0), \\
& d z(t):=\left(\begin{array}{c}
d q(t) \\
\dot{d} q(t),
\end{array}\right), \dot{d} q:=\frac{d}{d t} d q, \ddot{d} q:=\frac{d^{2}}{d t^{2}} d q,
\end{aligned}
$$

and the matrices $Y^{(j)}(t), K^{(j)}(t), D^{(j)}(t)$ and $M^{(j)}(t)$ are defined, cf. (76b-e), by

$$
\begin{aligned}
Y^{(j)}(t) & :=Y\left(q^{(j)}(t), \dot{q}^{(j)}(t), \ddot{q}^{(j)}(t)\right) \\
K^{(j)}(t) & :=K\left(\bar{p}_{D}^{(j)}, q^{(j)}(t), \dot{q}^{(j)}(t), \ddot{q}^{(j)}(t)\right) \\
D^{(j)} & :=D\left(\bar{p}_{D}^{(j)}, q^{(j)}(t), \dot{q}^{(j)}(t)\right), M^{(j)}(t):=M\left(\bar{p}_{D}^{(j)}, q^{(j)}(t)\right) .
\end{aligned}
$$


Local control corrections $d u=d u(t)$ stabilizing system (78a,b) can be obtained now by the following definition of the Jacobian of $\varphi^{(j)}(t, z)$ with respect to $z$ at $z=0$ :

$$
\begin{aligned}
\varphi_{z}^{(j)}(t, 0) & :=F_{z}\left(\bar{p}_{D}^{(j)}, q^{(j)}(t), \dot{q}^{(j)}(t), \ddot{q}^{(j)}(t)\right)-M^{(j)}(t)\left(K_{p}, K_{d}\right) \\
& =\left(K^{(j)}(t)-M^{(j)}(t) K_{p}, D^{(j)}(t)-M^{(j)}(t) K_{d}\right),
\end{aligned}
$$

where $K_{p}=\left(\gamma_{p k} \delta_{k \nu}\right), K_{d}=\left(\gamma_{d k} \delta_{k \nu}\right)$ are positive definite diagonal matrices with positive diagonal elements $\gamma_{p k}, \gamma_{d k}>0, k=1, \ldots, n$.

Inserting (79) into (78a), and assuming that $M^{(j)}=M^{(j)}(t)$ is regular for $t \geq t_{j}$, we find the following linear system of 2 nd order differential equations for $d q=d q(t)$

$$
\begin{aligned}
& \ddot{d} q(t)+K_{d} \dot{d} q(t)+K_{p} d q(t)=-M^{(j)}(t)^{-1} Y^{(j)}(t) \Delta p_{D}, t \geq t_{j}, \\
& d q\left(t_{j}\right)=\Delta q_{j}, \dot{d} q\left(t_{j}\right)=\dot{\Delta} q_{j} .
\end{aligned}
$$

Considering the right hand side of (80a), according to (78e), (76e) and (73d) we have that

$$
\begin{aligned}
Y^{(j)}(t) \Delta p_{D} & =Y\left(q^{(j)}(t), \dot{q}^{(j)}(t), \ddot{q}^{(j)}(t)\right) \Delta p_{D}=F\left(\Delta p_{D}, q^{(j)}(t), \dot{q}^{(j)}(t), \ddot{q}^{(j)}(t)\right) \\
& =M\left(\Delta p_{D}, q^{(j)}(t)\right) \ddot{q}^{(j)}(t)+h\left(\Delta p_{D}, q^{(j)}(t), \dot{q}^{(j)}(t)\right)
\end{aligned}
$$

Using then definition $(49 \mathrm{a}, \mathrm{b})$ of $q^{(j)}(t)$ and the representation $(18 \mathrm{a}, \mathrm{b})$ of $\dot{q}^{(j)}(t), \ddot{q}^{(j)}(t)$, we get

$$
\begin{aligned}
& Y^{(j)}(t) \Delta p_{D}=M\left(\Delta p_{D}, q_{e}^{(j)}\left(s^{(j)}(t)\right)\right)\left(q_{e}^{(j)^{\prime}}\left(s^{(j)}(t)\right) \frac{1}{2} \beta^{(j)}\left(s^{(j)}(t)\right)\right. \\
& \left.+q_{e}^{(j)^{\prime \prime}}\left(s^{(j)}(t)\right) \beta^{(j)}\left(s^{(j)}(t)\right)\right)+h\left(\Delta p_{D}, q_{e}^{(j)}\left(s^{(j)}(t)\right), q_{e}^{(j)^{\prime}}\left(s^{(j)}(t)\right) \sqrt{\beta^{(j)}\left(s^{(j)}(t)\right)}\right) .
\end{aligned}
$$

From (19b) we obtain now the following important representations, where we suppose that the feedforward control $u^{(j)}(t), t \geq t_{j}$, is given by $(50 \mathrm{c}, \mathrm{d})$.

Lemma 6.1. The following representations hold:
a) $Y^{(j)}(t) \Delta p_{D}=u_{e}\left(\Delta p_{D}, s^{(j)}(t) ; q_{e}^{(j)}(\cdot), \beta^{(j)}(\cdot)\right), t \geq t_{j}$;
b) $u^{(j)}(t)=u_{e}\left(\bar{p}_{D}^{(j)}, s^{(j)}(t) ; q_{e}^{(j)}(\cdot), \beta^{(j)}(\cdot)\right), t \geq t_{j}$;
c) $u^{(j)}(t)+Y^{(j)}(t) \Delta p_{D}=u_{e}\left(p_{D}, s^{(j)}(t) ; q_{e}^{(j)}(\cdot), \beta^{(j)}(\cdot)\right), t \geq t_{j}$. 
Proof. The first equation follows from (81a) and (19b). Equations (50c), $(18 \mathrm{a}, \mathrm{b})$ and $(19 \mathrm{~b})$ yield $(82 \mathrm{~b})$. Finally, (82c) follows from $(82 \mathrm{a}, \mathrm{b})$ and the linear parametrization of robots, cf. Remark 2.2.

Remark 6.1. Note that according to the transformation (19a) of the dynamic equation onto the $s$-domain, for the control input $u(t)$ we have the representation

$$
\begin{aligned}
u(t) & =u_{e}\left(p_{D}, s ; q_{e}(\cdot), \beta(\cdot)\right) \\
& =u_{e}\left(\bar{p}_{D}^{(j)}, s ; q_{e}(\cdot), \beta(\cdot)\right)+u_{e}\left(\Delta p_{D}, s ; q_{e}(\cdot), \beta(\cdot)\right)
\end{aligned}
$$

with $s=s(t)$.

Using (78d), it is easy to see that $(80 \mathrm{a}, \mathrm{b})$ can be described also by the 1 st order initial value problem

$$
\begin{aligned}
& \dot{d} z(t)=A d z(t)+\left(\begin{array}{l}
0 \\
\psi^{(j, 1)}(t)
\end{array}\right), t \geq t_{j} \\
& d z\left(t_{j}\right)=\Delta z_{j}=\left(\begin{array}{c}
q_{j}-\bar{q}_{j} \\
\dot{q}_{j}-\overline{\dot{q}}_{j}
\end{array}\right),
\end{aligned}
$$

where

$$
A:=\left(\begin{array}{cc}
0 & I \\
-K_{p}-K_{d}
\end{array}\right)
$$

and $\psi^{(j, 1)}(t)$ is defined, cf. (82a), by

$$
\begin{aligned}
\psi^{(j, 1)}(t) & :=-M^{(j)}(t)^{-1} Y^{(j)}(t) \Delta p_{D} \\
& =-M^{(j)}(t)^{-1} u_{e}\left(\Delta p_{D}, s^{(j)}(t) ; q_{e}^{(j)}(\cdot), \beta^{(j)}(\cdot)\right) .
\end{aligned}
$$

Consequently, for the first order expansion term $d z(t)$ of the deviation $\Delta z^{(j)}(t)$ between the actual state $z(t)=\left(\begin{array}{c}q(t) \\ \dot{q}(t)\end{array}\right)$ and the prescribed state $z^{(j)}(t)=$ $\left(\begin{array}{c}q^{(j)}(t) \\ \dot{q}^{(j)}(t)\end{array}\right), t \geq t_{j}$, we have the representation

$$
d z(t)=d z^{(j)}(t)=e^{A\left(t-t_{j}\right)} \Delta z_{j}+\int_{t_{j}}^{t} e^{A(t-\tau)}\left(\begin{array}{l}
0 \\
\psi^{(j, 1)}(\tau)
\end{array}\right) d \tau .
$$


Because of $E\left(\Delta p_{D}(\omega) \mid \mathcal{A}_{t_{j}}\right)=0$, we have that

$$
\begin{aligned}
E\left(\psi^{(j, 1)}(t) \mid \mathcal{A}_{t_{j}}\right) & =0, \\
E\left(d z(t) \mid \mathcal{A}_{t_{j}}\right) & =e^{A\left(t-t_{j}\right)} E\left(\Delta z_{j} \mid \mathcal{A}_{t_{j}}\right), t \geq t_{j},
\end{aligned}
$$

where, see (44a,b),

$$
E\left(\Delta z_{j} \mid \mathcal{A}_{t_{j}}\right)=E\left(z\left(t_{j}\right) \mid \mathcal{A}_{t_{j}}\right)-\bar{z}_{j}
$$

It is easy to see that the diagonal elements $\gamma_{d k}, \gamma_{p k}>0, k=1, \ldots, n$, of the positive definite diagonal matrices $K_{d}, K_{p}$, rep., can be chosen such that the fundamental matrix $\Phi(t, \tau)=e^{A(t-\tau)}, t \geq \tau$, is exponentially stable, i.e.

$$
\|\Phi(t, \tau)\| \leq a_{0} e^{-\lambda_{0}(t-\tau)}, t \geq \tau,
$$

with positive constants $a_{0}, \lambda_{0}$. A sufficient condition for (85a) reads

$$
\gamma_{d k}, \gamma_{p k}>0, k=1, \ldots, n \text {, and } \gamma_{d k}>2 \text { in case of double eigenvalues of } A \text {. }
$$

Define the generalized variance $\operatorname{var}\left(Z \mid \mathcal{A}_{t_{j}}\right)$ of a random vector $Z=Z(\omega)$ given $\mathcal{A}_{t_{j}}$ by $\operatorname{var}\left(Z \mid \mathcal{A}_{t_{j}}\right):=E\left(\left\|Z-E\left(Z \mid \mathcal{A}_{t_{j}}\right)\right\|^{2} \mid \mathcal{A}_{t_{j}}\right)$, and let $\sigma_{Z}^{(j)}:=\sqrt{\operatorname{var}\left(Z \mid \mathcal{A}_{t_{j}}\right)}$. Then, for the behaviour of the 1 st order error term $d z(t), t \geq t_{j}$, we have the following result:

Theorem 6.1. Suppose that the diagonal matrices $K_{d}, K_{p}$ are selected such that (85a) holds. Moreover, apply the local (i.e. first order) control correction

$$
d u(t):=\varphi_{z}^{(j)}(t, 0) d z(t),
$$

where $\varphi_{z}^{(j)}(t, 0)$ is defined by (79). Then, the following relations hold:

a) Asymptotic local stability in the mean:

$$
E\left(d z(t) \mid \mathcal{A}_{t_{j}}\right) \rightarrow 0, t \rightarrow \infty
$$

b) Mean absolute 1st order tracking error:

$$
\begin{array}{r}
E\left(\|d z\| \mid \mathcal{A}_{t_{j}}\right) \leq a_{0} e^{-\lambda_{0}\left(t-t_{j}\right)} \sqrt{\sigma_{z\left(t_{j}\right)}^{(j)^{2}}+\left\|E\left(z\left(t_{j}\right) \mid \mathcal{A}_{t_{j}}\right)-\bar{z}_{j}\right\|^{2}} \\
+a_{0} \int_{t_{j}}^{t} e^{-\lambda_{0}(t-\tau)} \sqrt{E\left(\left\|\psi^{(j, 1)}(\tau)\right\|^{2} \mid \mathcal{A}_{t_{j}}\right)} d \tau, t \geq t_{j}
\end{array}
$$


where

$$
\begin{aligned}
E\left(\left\|\psi^{(j, 1)}(t)\right\|^{2} \mid \mathcal{A}_{t_{j}}\right) & \leq\left\|M^{(j)}(t)^{-1}\right\|^{2} \sigma_{u_{e}}^{(j) 2}\left(s^{(j)}(t)\right), \\
\sigma_{u_{e}}^{(j)^{2}}\left(s^{(j)}(t)\right) & \leq\left\|Y^{(j)}(t)\right\|^{2} \operatorname{var}\left(p_{D}(\cdot) \mid \mathcal{A}_{t_{j}}\right)
\end{aligned}
$$

with

$$
\sigma_{u_{e}}^{(j)}(s):=\sqrt{\operatorname{var}\left(u_{e}\left(p_{D}(\cdot), s ; q_{e}^{(j)}(\cdot), \beta^{(j)}(\cdot)\right) \mid \mathcal{A}_{t_{j}}\right)} .
$$

Proof. Follows from (84a), (82a-d) and the fact that by Jensen's inequality $E \sqrt{X(\omega)} \leq \sqrt{E X(\omega)}$ for a nonnegative random variable $X=X(\omega)$.

Note that $\sigma_{u_{e}}^{(j) 2}\left(s^{(j)}(t)\right)$ can be interpreted as the risk of the feedforward control $u^{(j)}(t), t \geq t_{j} . \quad$ Using (69b), (78g), (86d,e) and changing then variables $\tau \rightarrow s$ in the integral in (86c), we obtain the following result:

Theorem 6.2. Under the assumptions of Theorem 6.1 , the following inequality holds for $t_{j} \leq t \leq t_{j}^{(j)}$ :

$$
\begin{aligned}
& E\left(\|d z(t)\| \mid \mathcal{A}_{t_{j}}\right) \leq a_{0} e^{-\lambda_{0}\left(t-t_{j}\right)} \sqrt{\sigma_{z\left(t_{j}\right)}^{(j)^{2}}+\left\|E\left(z\left(t_{j}\right) \mid \mathcal{A}_{t_{j}}\right)-\bar{z}_{j}\right\|^{2}} \\
&+\int_{s_{j}}^{s^{(j)}(t)} \frac{a_{0} e^{-\lambda_{0}\left(t-t^{(j)}(s)\right)}\left\|M\left(\bar{p}_{D}^{(j)}, q_{e}^{(j)}(s)\right)^{-1}\right\|}{\sqrt{\beta^{(j)}(s)}} \sigma_{u_{e}}^{(j)}(s) d s . \text { (87a) }
\end{aligned}
$$

The minimality or boundedness of the right hand side of $(87 \mathrm{a})$, hence, the robustness [11] of the present control scheme, is shown next:

Corollary 6.1 The meaning of the above inequality (87a) follows from the following important minimality/boundedness properties depending on the chosen substitute problem in (OSTP) for the trajectory planning problem under stochastic uncertainty:

i) The error contribution of the initial value $\bar{z}_{j}$ takes a minimum for $\bar{z}_{j}:=$ $E\left(z\left(t_{j}\right) \mid \mathcal{A}_{t_{j}}\right)$, cf. $(44 \mathrm{a}, \mathrm{b})$.

ii) The factor $\lambda_{0}$ can be increased by an appropriate selection of the matrices $K_{p}, K_{d}$ 
iii)

$$
\underline{c}_{M} \leq\left\|M\left(p_{D}^{(j)}, q_{e}^{(j)}(s)^{-1}\right)\right\| \leq \bar{c}_{M}, s_{j} \leq s \leq s_{f},
$$

with positive constants $\underline{c}_{M}, \bar{c}_{M}>0$. This follows from the fact that the mass matrix is always positive definite.

iv)

$$
\int_{s_{j}}^{s^{(j)}(t)} \frac{d s}{\sqrt{\beta^{(j)}(s)}} \leq \int_{s_{j}}^{s_{f}} \frac{d s}{\sqrt{\beta^{(j)}(s)}}=t_{f}^{(j)}-t_{f},
$$

where according to (OSTP), for minimum-time and related substitute problems, the right hand side is a minimum.

v) Depending on the chosen substitute problem in (OSTP), the generalized variance $\sigma_{u_{e}}^{(j)}(s), s_{f} \leq s \leq s_{f}$, is bounded pointwise by an appropriate upper risk level or minimized in a certain weighted mean sense.

For the minimality or boundedness of the generalized variance $\sigma_{u_{e}}^{(j)^{2}}(s), s_{j} \leq s \leq$ $s_{f}$, mentioned above, we give the following examples:

Working with the probabilistic control constraints (30a) and assuming that the vectors $u^{c}$ and $\rho_{u}$ are fixed, see (30d), according to (30f) we find that (30a) can be guaranteed by

$$
\sigma_{u_{e}}^{(j)^{2}}(s)+\left\|\bar{u}_{e}^{(j)}(s)-u^{c}\right\|^{2} \leq\left(1-\alpha_{u}\right) \min _{1 \leq k \leq n} \rho_{u k}^{2}, s_{j} \leq s \leq s_{f}
$$

where $\bar{u}_{e}^{(j)}(s):=u_{e}\left(\bar{p}_{D}^{(j)}, s ; q_{e}^{(j)}(\cdot), \beta^{(j)}(\cdot)\right)$. Hence, with $(87 \mathrm{~d})$ we have then the condition

$$
\sigma_{u_{e}}^{(j)^{2}}(s) \leq\left(1-\alpha_{u}\right) \min _{1 \leq k \leq n} \rho_{u_{e}}^{2}, s_{f} \leq s \leq s_{f},
$$

cf. (87a). Under special distribution assumptions for $p_{D}(\omega)$ more exact explicit deterministic conditions for (30a) may be derived, see Remark 2.2.

If minimum force and moment should be achieved along the trajectory, hence, if $\phi=0$ and $L=\|u(t)\|^{2}$, see (6), then, according to substitute problem (29a-f, f') we have the following minimality property:

$$
\int_{s_{j}}^{s_{f}}\left(\sigma_{u_{e}}^{(j)^{2}}(s)+\left\|\bar{u}_{e}^{(j)}(s)\right\|^{2}\right) \frac{d s}{\sqrt{\beta^{(j)}(s)}}=\min _{q_{e}(\cdot), \beta(\cdot)} E\left(\int_{t_{j}}^{t_{f}}\|u(t)\|^{2} d t \mid \mathcal{A}_{t_{j}}\right) .
$$


Mean/variance condition for $u_{e}$ : Condition (29b) in substitute problemd (29a-f, $\left.\mathrm{f}^{\prime}\right)$ may read in case of fixed bounds $u^{\min }, u^{\max }$ for the control $u(t)$ as follows:

$$
\begin{aligned}
u^{\min } & \leq u_{e}\left(\bar{p}_{D}^{(j)}, s ; q_{e}(\cdot), \beta(\cdot)\right) \leq u^{\max }, s_{j} \leq s \leq s_{f} \\
\sigma_{u_{e}}^{(j)}(s) & \leq \sigma_{u_{e}}^{\max }, s_{f} \leq s \leq s_{f}
\end{aligned}
$$

with a given upper bound $\sigma_{u_{e}}^{\max }$, cf. (87d').

According to the above Theorem 6.2, further stability results, especially the convergence

$$
E\left(\|d z(t)\| \mid \mathcal{A}_{t_{j}}\right) \rightarrow 0 \text { for } j \rightarrow \infty, t \rightarrow \infty
$$

of the mean absolute first order tracking error can be obtained if, by using a suitable update law $[1,2,4,10]$ for the parameter estimates, hence, for the a posteriori distribution $P\left(\cdot \mid \mathcal{A}_{t_{j}}\right)$, we have that, see (86f),

$$
\operatorname{var}\left(p_{D}(\cdot) \mid \mathcal{A}_{t_{j}}\right)=\operatorname{var}\left(p_{D}(\cdot) \mid \mathcal{A}_{t_{j}}\right)(t) \rightarrow 0 \text { for } j \rightarrow \infty, t \rightarrow \infty
$$

\subsection{The 2nd order differential $d^{2} q$}

In order to derive a representation of the second order differential $d^{2} q$, equation (77a) for $\frac{\partial q}{\partial \epsilon}(t, \epsilon)$ is represented as follows:

$$
F_{p_{D}} \Delta p_{D}+F_{z} \frac{\partial z}{\partial \epsilon}+F_{\ddot{q}} \frac{\partial \ddot{q}}{\partial \epsilon}=\frac{\partial u}{\partial \epsilon}=\varphi_{z}^{(j)} \frac{\partial z}{\partial \epsilon},
$$

where $F=F\left(p_{D}, z, \ddot{q}\right), z=\left(\begin{array}{l}q \\ \dot{q}\end{array}\right)$, is given by $(73 \mathrm{~d})$, see also $(76 \mathrm{e})$, and therefore

$$
\begin{aligned}
F_{p_{D}} & =F_{p_{D}}(q, \dot{q}, \ddot{q})=Y(q, \dot{q}, \ddot{q}), F_{\ddot{q}}=F_{\ddot{q}}\left(p_{D}, q\right)=M\left(p_{D}, q\right) \\
F_{z} & =F_{z}\left(p_{D}, q, \dot{q}, \ddot{q}\right)=\left(F_{q}, F_{\dot{q}}\right)=\left(K\left(p_{D}, q, \dot{q}, \ddot{q}\right), D\left(p_{D}, q, \dot{q}\right)\right) .
\end{aligned}
$$

Moreover, we have that

$$
\varphi^{(j)}=\varphi^{(j)}\left(t, z-z^{(j)}(t)\right), z=\left(\begin{array}{c}
q(t, \epsilon) \\
\dot{q}(t, \epsilon)
\end{array}\right), p_{D}=p_{D}(\epsilon) .
$$

By differentiation of (89a) with respect to $\epsilon$, we obtain

$$
2 F_{p_{D} z} \cdot\left(\Delta p_{D}, \frac{\partial z}{\partial \epsilon}\right)+2 F_{p_{D} \ddot{q}} \cdot\left(\Delta p_{D}, \frac{\partial \ddot{q}}{\partial \epsilon}\right)+F_{z z} \cdot\left(\frac{\partial z}{\partial \epsilon}, \frac{\partial z}{\partial \epsilon}\right)+2 F_{z \ddot{q}} \cdot\left(\frac{\partial z}{\partial \epsilon}, \frac{\partial \ddot{q}}{\partial \epsilon}\right)
$$




$$
+F_{z} \frac{\partial^{2} z}{\partial \epsilon^{2}}+F_{\ddot{q}} \frac{\partial^{2} \ddot{q}}{\partial \epsilon^{2}}=\varphi_{z z}^{(j)} \cdot\left(\frac{\partial z}{\partial \epsilon}, \frac{\partial z}{\partial \epsilon}\right)+\varphi_{z}^{(j)} \frac{\partial^{2} z}{\partial \epsilon^{2}},
$$

with the 2nd order partial derivatives

$$
\begin{aligned}
F_{p_{D} z} & =F_{p_{D} z}(z, \ddot{q}), F_{p_{D} \ddot{q}}=F_{p_{D} \ddot{q}}(q)=M_{p_{D}}(q) \\
F_{z z} & =F_{z z}\left(p_{D}, z, \ddot{q}\right), F_{z \ddot{q}}=F_{z \ddot{q}}\left(p_{D}, z\right)=\left(M_{q}\left(p_{D}, q\right), 0\right) .
\end{aligned}
$$

Moreover, differentiation of (77b) with respect to $\epsilon$ yields the initial values

$$
\frac{\partial^{2} q}{\partial \epsilon^{2}}\left(t_{j}, \epsilon\right)=0, \frac{d}{d t} \frac{\partial^{2} q}{\partial \epsilon^{2}}\left(t_{j}, \epsilon\right)=\frac{\partial^{2} \dot{q}}{\partial \epsilon^{2}}\left(t_{j}, \epsilon\right)=0
$$

for $\frac{\partial^{2} z}{\partial \epsilon^{2}}=\frac{\partial^{2} z}{\partial \epsilon^{2}}(t, \epsilon)=\left(\frac{\partial^{2} q}{\partial \epsilon^{2}}(t, \epsilon), \frac{\partial^{2} \dot{q}}{\partial \epsilon^{2}}(t, \epsilon)\right), t \geq t_{j}, 0 \leq \epsilon \leq 1$.

Putting now $\epsilon=0$, from (90a) we obtain the following differential equation for the 2nd order differential $d^{2} q(t)=\frac{\partial^{2} q}{\partial \epsilon^{2}}(t, 0)$ of $q=q(t, \epsilon)$ :

$$
\begin{aligned}
& K^{(j)}(t) d^{2} q(t)+D^{(j)}(t) \frac{d}{d t} d^{2} q(t)+M^{(j)}(t) \frac{d^{2}}{d t^{2}} d^{2} q(t) \\
+ & \left(F_{z z}\left(\bar{p}_{D}^{(j)}, q^{(j)}(t), \dot{q}^{(j)}(t), \ddot{q}^{(j)}(t)\right)-\varphi_{z z}^{(j)}(t, 0)\right) \cdot(d z(t), d z(t))-\varphi_{z}^{(j)}(t, 0) d^{2} z(t) \\
=- & 2\left(F_{p_{D} z}^{(j)}(t) \cdot\left(\Delta p_{D}, d z(t)\right)+F_{p_{D}}^{(j)}(t) \cdot\left(\Delta p_{D}, \ddot{d} q(t)\right)+F_{z \ddot{q}}^{(j)}(t) \cdot(d z(t), \ddot{d} q(t)) .\right.
\end{aligned}
$$

Here, we set

$$
d^{2} z(t):=\left(\begin{array}{c}
d^{2} q(t) \\
\frac{d}{d t} d^{2} q(t)
\end{array}\right),
$$

and the vectorial Hessians $F_{p_{D} z}^{(j)}(t), F_{p_{D} \ddot{q}}^{(j)}(t), F_{z \ddot{q}}^{(j)}(t)$ follow from $(90 \mathrm{~b})$ by inserting there the argument $\left(p_{D}, q, \dot{q}, \ddot{q}\right):=\left(\bar{p}_{D}^{(j)}, q^{(j)}(t), \dot{q}^{(j)}(t), \ddot{q}^{(j)}(t)\right)$. Furthermore, (90d) yields the following initial condition for $d^{2} q(t)$

$$
d^{2} q\left(t_{j}\right)=0, \frac{d}{d t} d^{2} q\left(t_{j}\right)=0 .
$$

According to (91a) we define now, cf. (79), the second order derivative of $\varphi^{(j)}$ with respect to $z$ at $\Delta z=0$ by

$$
\varphi_{z z}^{(j)}(t, 0):=F_{z z}\left(\bar{p}_{D}^{(j)}, q^{(j)}(t), \dot{q}^{(j)}(t), \ddot{q}^{(j)}(t)\right), t \geq t_{j} .
$$

Using then definition (77) of the Jacobian of $\varphi^{(j)}$ with respect to $z$ at $\Delta z=0$, for $d^{2} q=d^{2} q(t), t \geq t_{j}$, we find the following initial value problem

$$
\frac{d^{2}}{d t^{2}} d^{2} q(t)+K_{d} \frac{d}{d t} d^{2} q(t)+K_{p} d^{2} q(t)
$$




$$
\begin{aligned}
& =-M^{(j)}(t)^{-1}{\widetilde{\mathbf{D}^{2} F}}^{(j)}(t) \cdot\left(\Delta p_{D}, d z(t), \ddot{d} q(t)\right)^{2}, t \geq t_{j}, \\
d^{2} q\left(t_{j}\right) & =0, \frac{d}{d t} d^{2} q\left(t_{j}\right)=0
\end{aligned}
$$

where the sub-Hessian

$$
\widetilde{\mathbf{D}^{2} F^{(j)}}(t):=\widetilde{\mathbf{D}^{2} F}\left(\bar{p}_{D}^{(j)}, q^{(j)}(t), \dot{q}^{(j)}(t), \ddot{q}^{(j)}(t)\right)
$$

of $F$ results from the Hessian of $F$ by replacing the diagonal block $F_{z z}$ by zero. Of course, we have that, cf. (91a),

$$
\begin{array}{r}
{\widetilde{\nabla^{2} F}}^{(j)} \cdot\left(\Delta_{p_{D}}, d z(t), \ddot{d} q(t)\right)^{2}=2\left(F_{p_{D} z}^{(j)}(t) \cdot\left(\Delta p_{D}, d z(t)\right)\right. \\
\left.+F_{p_{D} \ddot{q}}^{(j)}(t) \cdot\left(\Delta p_{D}, \ddot{d} q(t)\right)+F_{z \ddot{q}}^{(j)}(t) \cdot(\ddot{d} q(t), d z(t))\right) .
\end{array}
$$

Comparing now the initial value problems $(80 \mathrm{a}, \mathrm{b})$ and $(93 \mathrm{a}, \mathrm{b})$ for the first and second order differential of $q=q(t, \epsilon)$, we recognize that the linear 2 nd order differential equations have - up to the right hand side - exactly the same form.

According to (83a-d) and (84a) we know that the 1st order expansion terms

$$
(d z(t), \ddot{d} q(t))=(d q(t), \dot{d} q(t), \ddot{d} q(t)), t \geq t_{j},
$$

in the tracking error depend linearly on the error term

$$
\Delta \theta^{(j)}=\Delta \theta^{(j)}(t):=\left(\begin{array}{c}
e^{A\left(t-t_{j}\right)} \Delta z_{j} \\
\Delta p_{D}
\end{array}\right)\left(\rightarrow\left(\begin{array}{c}
0 \\
\Delta p_{D}
\end{array}\right), t \rightarrow \infty\right)
$$

corresponding to the variations/disturbances of the initial values $\left(q_{j}, \dot{q}_{j}\right)$ and dynamic parameters $p_{D}$. Consequently, we have this observation:

Lemma 6.2 The right hand side

$$
\psi^{(j, 2)}(t):=-M^{(j)}(t)^{-1}{\widetilde{\mathbf{D}^{2} F}}^{(j)}(t) \cdot\left(\Delta p_{D}, d z(t), \ddot{d} q(t)\right)^{2}, t \geq t_{j},
$$

of the error differential equation (93a) for $d^{2} q(t)$ is quadratic in the error term $\Delta \theta^{(j)}(\cdot)$.

According to (93a-c), the second order expansion term

$$
d^{2} z(t)=\left(d^{2} q(t), \frac{d}{d t} d^{2} q(t)\right), t \geq t_{j},
$$

of the Taylor expansion of the tracking error can be represented again by the solution of the system of linear differential equations (83a-c), where now $\psi^{(j, 1)}(t)$ 
is replaced by $\psi^{(j, 2)}(t)$ defined by $(95)$, and the initial values are given by $d^{2} z\left(t_{j}\right)=$ 0 . Thus, applying again solution formula (84a), we find

$$
d^{2} z(t)=\int_{t_{j}}^{t} e^{A(t-\tau)}\left(\begin{array}{c}
0 \\
\psi^{(j, 2)}(\tau)
\end{array}\right) d \tau .
$$

From (94)-(96) and Lemma 6.2 we get now the following result:

Theorem 6.3. The second order tracking error expansion terms $\left(d^{2} z(t), \frac{d^{2}}{d t^{2}} d^{2} q(t)\right)=$ $\left(d^{2} q(t), \frac{d}{d t} d^{2} q(t), \frac{d^{2}}{d t^{2}} d^{2} q(t)\right), t \geq t_{j}$, depend

i) quadratically on the 1 st order error terms $\left(\Delta p_{D}, d z(t), \ddot{d} q(t)\right)$ and

ii) quadratically on the error term $\Delta \theta^{(j)}(\cdot)$ corresponding to the variations/disturbances of the initial values and dynamics parameters.

Because of (96), the stability properties of the second order tracking error expansion term $d^{2} q(t), t \geq t_{j}$, are determined again by the matrix exponential function $\Phi(t, \tau)=e^{A(t-\tau)}$ and the remainder $\psi^{(j, 2)}(t)$ given by $(95)$.

According to Theorem 6.2 and Corollary 6.1 we know that the disturbance term $\psi^{(j, 1)}(t)$ of (80a), (83a) is reduced directly by control-constraints (for $u_{e}$ ) present in (OSTP). Concerning the next disturbance term $\psi^{(j, 2)}(t)$ of $(93 \mathrm{a})$, by (95) we note first that a reduction of the 1st order error terms $\left(\Delta p_{D}, d z(\cdot), \ddot{d} q(\cdot)\right)$ yields a reduction of $\psi^{(j, 2)}$ and by (96) also a reduction of $d^{2} z(t), \frac{d^{2}}{d t^{2}} d^{2} q(t), t \geq t_{j}$. Comparing then definitions (83d) and (95) of the disturbances $\psi^{(j, 1)}, \psi^{(j, 2)}$, we observe that, corresponding to $\psi^{(j, 1)}$, certain terms in $\psi^{(j, 2)}$ depend only on the reference trajectory $q^{(j)}(t), t \geq t_{j}$, of stage $j$. Hence, this observation yields the following result.

Theorem 6.4. The disturbance $\psi^{(j, 2)}$ of (93a), and consequently also the second order tracking error expansion terms $d^{2} q(t), \frac{d}{d t} d^{2} q(t), \frac{d^{2}}{d t^{2}} d^{2} q(t), t \geq t_{j}$, can be diminished by

i) reducing the 1 st order error terms $\left(\Delta p_{D}, d z(\cdot), \ddot{q}(\cdot)\right)$, and by

ii) taking into (OSTP) additional conditions for the unknown functions $q_{e}(s), \beta(s), s_{j} \leq s \leq s_{f}$, guaranteeing that (the norm of) the sub-Hessian $\widetilde{\mathbf{D}^{2} F^{(j)}}(t), t \geq t_{j}$, fulfills a certain minimality or boundedness condition. 
Proof. Follows from definition (95) of $\psi^{(j, 2)}$ and representation (96) of the second order tracking error expansion term $d^{2} z$.

\subsection{3rd and higher order differentials}

By further differentiation of equations $(90 \mathrm{a}, \mathrm{d})$ with respect to $\epsilon$ and by putting $\epsilon=0$, also the 3 rd and higher order differentials $d^{l} q(t), t \geq t_{j}, l \geq 3$, con be obtained. We observe that the basic structure of the differential equations for the differentials $d^{l} q, l \geq 1$, remains the same. Hence, by induction for the differentials $d^{l} z, l \geq 1$, we have the following representation:

Theorem 6.5. Defining the tensorial coefficients of the Taylor expansion (75d) for the feedback control law $\varphi^{(j)}=\varphi^{(j)}(t, \Delta z)$ by

$$
D_{z}^{l} \varphi^{(j)}(t, 0):=D_{z}^{l} F\left(\bar{p}_{D}^{(j)}, q^{(j)}(t), \dot{q}^{(j)}(t), \ddot{q}^{(j)}(t)\right), t \geq t_{j}, l=1,2, \ldots,
$$

the differentials $d^{l} z(t)=\left(d^{l} q(t), \frac{d}{d t} d^{l} q(t)\right), t \geq t_{j}$, may be represented by the systems of linear differential equations

$$
\frac{d}{d t} d^{l} z(t)=A d^{l} z(t)+\left(\begin{array}{c}
0 \\
\psi^{(j, l)}(t)
\end{array}\right), t \geq t_{j}, l=1,2, \ldots,
$$

with the same system matrix $A$ and the disturbance terms $\psi^{(j, l)}(t), t \geq t_{j}$, given by

$$
\begin{array}{r}
\psi^{(j, l)}(t)=-M^{(j)}(t)^{-1} \pi\left(\widetilde{\mathbf{D}^{\lambda} F^{(j)}}(t), 2 \leq \lambda \leq l ; \Delta p_{D}, d^{j} z(t),\right. \\
\left.\frac{d^{2}}{d t^{2}} d^{k} q(t), 1 \leq j, k \leq l-1\right), l \geq 2,
\end{array}
$$

where $\pi$ is a polynomial in the variables $\Delta p_{D}$ and $d^{j} z(t), \frac{d^{2}}{d t^{2}} d^{k} q(t), 1 \leq j, k \leq$ $l-1$, having coefficients from the sub-operators $\widetilde{\mathbf{D}^{\lambda} F}(t)$ of $\mathbf{D}^{\lambda} F^{(j)}(t)$ containing mixed partial derivatives of $F$ with respect to $\Delta p_{D}, z, \ddot{q}$ of order $\lambda=2,3, \ldots, l-1$ at $\left(p_{D}, q, \dot{q}, \ddot{q}\right)=\left({\overline{p_{D}}}^{(j)}, q^{(j)}(t), \dot{q}^{(j)}(t), \ddot{q}^{(j)}(t)\right)$ such that the disturbance $\psi^{(j, l)}$ is a polynomial of order $l$ with respect to the error term $\Delta \theta^{(j)}(\cdot)$.

According to (73d), (4a-d) and Remark 2.2 we know that the vector function $F=F\left(p_{D}, q, \dot{q}, \ddot{q}\right)$ is linear in $p_{D}$, linear in $\ddot{q}$ and quadratic in $\dot{q}$ (supposing 
case 4c)) and analytical with respect to $q$. Hence, working with a polynomial approximation with respect to $q$, we may assume that

$$
\mathbf{D}^{l} F^{(j)}(t)=\mathbf{D}^{l} F\left(\bar{p}_{D}^{(j)}, q^{(j)}(t), \dot{q}^{(j)}(t), \ddot{q}^{(j)}(t)\right) \approx 0, t \geq t_{j}, l \geq l_{0}
$$

for some index $l_{0}$.

According to the expansion (75d) of the feedback control law $\varphi^{(j)}=\varphi^{(j)}(t, \Delta z)$, definition (97) of the corresponding coefficients and Theorem 6.4 we have now this robustness [11] result:

Theorem 6.6. The Taylor expansion $(75 \mathrm{~d})$ of the feedback control law $\varphi^{(j)}=$ $\varphi^{(j)}(t, \Delta z)$ stops after a finite number $\left(\leq l_{0}\right)$ of terms. Besides the conditions for $u_{e}$ contained automatically in (OSTP) via the control constraints, the mean absolute tracking error $E\left(\|\Delta z(t)\| \mid \mathcal{A}_{t_{j}}\right)$ can be diminished further be including additional conditions for the functions $\left(q_{e}(s), \beta(s)\right), s_{j} \leq s \leq s_{f}$, in (OSTP) which guarantee a minimality or boundedness condition for (the norm of) the sub-operators of mixed partial derivatives ${\widetilde{\mathbf{D}^{2} F}}^{(j)}(t), t \geq t_{j}, \lambda=2,3, \ldots, l_{0}$.

\section{Acknowledgement}

The author thanks Mrs. Elisabeth Lößl, UniBw München, for the excellent LaTex-setting of the manuskript.

\section{References}

[1] Arimoto, S.: Control Theory of Non-Linear Mechanical Systems. Oxford: Clarendon Press, 1996.

[2] Aström, K.J.; Wittenmark, B.: Adaptive Control. Reading, Mass. [etc.]: Addison-Wesley cop., 1995.

[3] Aurnhammer, A.; Marti, K.: OSTP/Fortran-Program For Optimal Stochastic Trajectory Planning of Robots. UniBw München, 2000

[4] Bastian, G. et al. (eds.): Theory of Robot Control. Berlin-Heidelberg-New York: SpringerVerlag, 1996

[5] Bernhardt, R.; Albright, S.L.: Robot Calibration. London [etc.]: Chapman and Hall, 1993

[6] Bobrow, J.E.; Dubowsky, S.; Gibson, J.S.: Time-Optimal Control of Robotic Manipulators Along Specified Paths. The Int. J. Of Robotics Research 4, No. 3, pp. 3-17 (1985)

[7] Bobrow, J.E.: Optimal Robot Path Planning Using the Minimum-Time Criterion. IEEE J. of Robotics and Automation 4, No. 4, pp. 443-450 (1988) 
[8] Bohlin, R.: Motion Planning for Industrial Robots. Goeteborg: Chalmers Univ., 1999

[9] Chen, Y.-Ch.: Solving Robot Trajectory Planning Problems with Uniform Cubic B-Splines. Optimal Control Applications and Methods 12, pp. 247-262 (1991)

[10] Craig, J.J.: Adaptive Control of Mechanical Manipulators. Reading Mass. (etc.): AddisonWesley Publ. Company, 1988

[11] Dullerud, G.E.; Paganini, F.: A Course in Robust Control Theory. New York: SpringerVerlag, 2000

[12] Gessner, P.; Spreman, K.: Optimierung in Funktionsenräumen. LNMES, Vol. 64. Berlin [etc.]: Springer-Verlag, 1972

[13] Haubach-Lippmann, C.: Stochastische Strukturoptimierung flexibler Roboter. Fortschrittberichte VDI, Reihe 8, Nr. 706. Düsseldorf: VDI Verlag 1998

[14] Holtgrewe, D.: Adaptive Regelung flexibler Roboter. Paderborn: Igel Verlag, 1996

[15] Hoppen, P.: Autonome mobile Roboter. Mannheim (etc.): B.I. Wissenschaftsverlag, 1992

[16] Hwang, Y.K.; Ahuja, N.: Gross motion planning - a survey. ACM Comp. Survey 24, No. 3, pp. 219-291 (1992)

[17] Isermann, R.: Identifikation dynamischer Systeme. Berling: Springer-Verlag, 1988

[18] Johanni, R.: Optimale Bahnplanung bei Industrierobotern. Fortschrittberichte VDI, Reihe 18, Nr. 51. Düsseldorf: VDI-Verlag, 1988

[19] Khalil, H.K.: Nonlinear Systems. New York (etc.): Maxmillan Publ. Comp., 1992

[20] Lin, K.: Zur adaptiven und langzeitprädikativen Regelung mit einem statistischen Optimierungskriterium. Dissertation, TU Hamburg-Harburg, 1997

[21] Marti, K.: Approximation stochastischer Optimierungsprobleme. Königstein/Ts.: Hain, 1979

[22] Marti, K.; Qu, S.: Optimal Trajectory Planning for Robots under the Consideration of Stochastic Parameters and Disturbances. J. of Intelligent and Robotic Systems 15, pp. 19-23 (1996)

[23] Marti, K.; Qu, S.: Path Planning for Robots by Stochastic Optimization Methods. J. of Intelligent and Robotic Systems 22, pp. 117-127 (1998)

[24] Marti, K.: Path Planning for Robots under Stochastic Uncertainty. Optimization 45, pp. 163-195 (1999)

[25] Marti, K., Qu, S.: Adaptive stochastic path planning for robots-Real-time optimization by means of neural networks. In: M.P. Polis et al. (eds.): Systems Modelling and Optimization, Proceed. 18th TC7 Conference. Boca Raton: Chapman and Hall/CRC Research Notes in Mathematics 1999, pp. 486-494

[26] Miesbach, S.: Bahnführung von Robotern mit Neuronalen Netzen. Dissertation, TU München, Fakultät für Mathematik, 1995

[27] Pai, D.K.; Leu, M.C.: Uncertainty and Compliance of Robot Manpulators with Application to Task Feasibility. The Int. J. of Robotic Research 10, Nor. 3, pp. 200-212 (1991)

[28] Pfeiffer, F.; Johanni, R.: A Concept for Manipulator Trajectory Planning. IEEE J. of Robotics and Automation, Vol. RA-3, No. 2, pp. 115-123 (1987) 
[29] Pfeiffer, F.; Reithmeier, E.: Roboterdynamik. Stuttgart: Teubner, 1987

[30] Pfeiffer, R.; Richter, K.: Optimal Path Planning Including Forces at the Gripper. J. of Intelligent and Robotic Systems 3, pp. 251-258 (1990)

[31] Qu, S.: Optimal Bahnplanung unter Berücksichtigung stochastischer Paramterschwankungen. Fortschrittberichte VDI, Reihe 8, Nr. 472. Düsseldorf: VDI-Verlag, 1995

[32] Qu, Z.; Dawson, D.M.: Robust Tracking Control of Robot Manipulators. New York: IEEE Press, 1996

[33] Redfern, D.A., Goh, C.J.: Feedback control of state constrained optimal control problems. In: J. Dolezal; J. Fidler (eds.): System Modelling and Optimization. London (etc.): Chapman and Hall, 1996, pp. 442-449

[34] Reif, K.: Steuerung von nichtlinearen Systemem mit Homotopie-Verfahren. Fortschrittberichte VDI, Reihe 8: Mess-, Steuerungs- und Regelungstechnik, Nr. 631. Düsseldorf: VDI Verlag, 1997

[35] Schilling, R.J.: Fundamentals of Robotics; Analysis and Control. London [etc.]: Prentice Hall, 1990

[36] Schlöder, J.P.: Numerische Methoden zur Behandlung hochdimensionaler Aufgaben der Parameteridentifizierung. Dissertation, Universität Bonn, Math.-Naturwissenschaftliche Fakultät, 1988

[37] Schröer, K.: Identifikation von Kalibrierungsparametern kinematischer Ketten. MünchenWien: Carl Hanser, 1993

[38] Sciavicco, L.; Siciliano, B.: Modelling and Control of Robot Manipulators. london [etc.]: Springer-Verlag, 2000

[39] Shin, K.G.; McKay, N.D.: Minimum-Time Control of Robotic Manipulators with Geometric Path Constraints. IEEE Trans. on Automatic Control, Vol. AC-30, pp. 531-541 (1985)

[40] Slotine, J.-J.; Li, W.: Applied Nonlinear Control. Englewood Cliffs, N.J.: Prentice-Hall Int., Inc., 1991

[41] Stengel, R.F.: Stochastic Optimal Control - Theory and Application. New York (etc.): J. Wiley, 1986

[42] Stone, H.W.: Kinematic Modeling, Identification, and Control of Robotic Manipulators. Boston [etc.]: Kluwer Acad. Publ., 1987

[43] Troch, I.: Time-Optimal Path Generation for Continuous and Quasi-Continuous Path Control of Industrial Robots. J. Intelligent and Robotic Systems 2, pp. 1-28 (1989)

[44] Weinmann, A.: Uncertain Models and Robust Control. Wien-New York: Springer-Verlag, 1991

[45] Weinzierl, K.: Konzepte zur Steuerung und Regelung nichtlinearer Systeme auf der Basis der Jacobi-Linearisierung. Aachen: Verlag Shaker, 1995 